THEORY OF COMPUTING, Volume 5 (2009), pp. 1-42

www.theoryofcomputing.org

\title{
The Power of Unentanglement
}

\author{
Scott Aaronson* Salman Beigi ${ }^{\dagger} \quad$ Andrew Drucker $^{\ddagger} \quad$ Bill Fefferman $^{\S}$ \\ Peter Shor ${ }^{\text {II }}$
}

Received: April 22, 2008; revised: December 27, 2008; published: May 11, 2009.

\begin{abstract}
The class QMA $(k)$, introduced by Kobayashi et al., consists of all languages that can be verified using $k$ unentangled quantum proofs. Many of the simplest questions about this class have remained embarrassingly open: for example, can we give any evidence that $k$ quantum proofs are more powerful than one? Does $\mathrm{QMA}(k)=\mathrm{QMA}(2)$ for $k \geq 2$ ? Can $\mathrm{QMA}(k)$ protocols be amplified to exponentially small error?
\end{abstract}

In this paper, we make progress on all of the above questions.

- We give a protocol by which a verifier can be convinced that a 3SAT formula of size $m$ is satisfiable, with constant soundness, given $\widetilde{O}(\sqrt{m})$ unentangled quantum witnesses with $O(\log m)$ qubits each. Our protocol relies on the existence of very short PCPs.

- We show that assuming a weak version of the Additivity Conjecture from quantum information theory, any $\mathrm{QMA}(2)$ protocol can be amplified to exponentially small error, and $\mathrm{QMA}(k)=$ $\mathrm{QMA}(2)$ for all $k \geq 2$.

- We prove the nonexistence of "perfect disentanglers" for simulating multiple Merlins with one.

\section{ACM Classification: F.1.2, F.1.3}

AMS Classification: 81P68, 68Q15, 68Q17

Key words and phrases: quantum computing, PCPs, entanglement, Merlin-Arthur, 3SAT

${ }^{*}$ To whom correspondence should be addressed. Supported by MIT as well as the NSF IGERT program.

† Supported in part by NSF Grant CCF-0829421

¥Supported by an Akamai Presidential Graduate Fellowship.

${ }^{\S}$ Supported by a Caltech Division of Engineering and Applied Science Fellowship and NSF Grants CCF-0830787, CCF0829909.

IS Supported in part by NSF Grant CCF-0829421 


\section{S. Aaronson, S. Beigi, A. Drucker, B. Fefferman, And P. Shor}

\section{Introduction}

Quantum entanglement is often described as a complicated, hard-to-understand resource. But ironically, many questions in quantum computing are easiest to answer assuming unlimited entanglement, and become much more difficult if entanglement is not allowed! One way to understand this is that Hilbert space - the space of all quantum states - has extremely useful linear-algebraic properties, and when we restrict to the set of separable states we lose many of those properties. So for example, finding a quantum state that maximizes the probability of a given measurement outcome is just a principal eigenvector problem, but finding a separable state that does the same is NP-hard [7].

These observations naturally give rise to a general question at the intersection of computational complexity and entanglement theory. Namely: supposing we had $k$ quantum proofs, could we use the promise that the proofs were unentangled to verify mathematical statements beyond what we could verify otherwise?

\subsection{Background and related work}

The class QMA, or Quantum Merlin-Arthur, consists of all languages that admit a proof protocol in which Merlin sends Arthur a polynomial-size quantum state $|\psi\rangle$, and then Arthur decides whether to accept or reject in quantum polynomial time. This class was introduced by Knill [17], Kitaev [15], and Watrous [29] as a quantum analogue of NP. By now we know a reasonable amount about QMA: for example, it allows amplification of success probabilities, is contained in PP, and has natural complete promise problems. (See Aharonov and Naveh [2] for a survey.)

In 2003, Kobayashi, Matsumoto, and Yamakami [19] defined a generalization of QMA called QMA $(k)$. Here there are $k$ Merlins, who send Arthur $k$ quantum proofs $\left|\psi_{1}\right\rangle, \ldots,\left|\psi_{k}\right\rangle$ respectively that are guaranteed to be unentangled with each other. (Thus QMA $(1)=$ QMA.) Notice that in the classical case, this generalization is completely uninteresting: we have $\mathrm{MA}(k)=\mathrm{MA}$ for all $k$, since we can always simulate $k$ Merlins by a single Merlin who sends Arthur a concatenation of the $k$ proofs. In the quantum case, however, a single Merlin could cheat by entangling the $k$ proofs, and we know of no general way to detect such entanglement.

When we try to understand QMA $(k)$, we encounter at least three basic questions. First, do multiple quantum proofs ever actually help? That is, can we find some sort of evidence that $\mathrm{QMA}(k) \neq \mathrm{QMA}(1)$ for some $k$ ? Second, can $\mathrm{QMA}(k)$ protocols be amplified to exponentially small error? Third, are two Merlins the most we ever need? That is, does $\mathrm{QMA}(k)=\mathrm{QMA}(2)$ for all $k \geq 2$ ? $^{1}$

We know of three previous results that are relevant to the above questions.

First, in their original paper on QMA $(k)$, Kobayashi et al. [19] proved that a positive answer to the second question implies a positive answer to the third. That is, if $\mathrm{QMA}(k)$ protocols can be amplified, then $\mathrm{QMA}(k)=\mathrm{QMA}(2)$ for all $k \geq 2$.

Second, Liu, Christandl, and Verstraete [21] gave a natural problem from quantum chemistry, called pure state N-representability, which is in QMA(2) but is not known to be in QMA.

\footnotetext{
${ }^{1}$ The second and third questions are motivated, in part, by an analogy to classical multi-prover interactive proof systems — where the Parallel Repetition Theorem of Raz [26] and the $\operatorname{MIP}(k)=\operatorname{MIP}(2)$ theorem of Ben-Or et al. [3] turned out to be crucial for understanding the class MIP.
} 


\section{The Power of Unentanglement}

Third, Blier and Tapp [7] recently (and independently of us) gave an interesting QMA(2) protocol for an NP-complete problem, namely 3-COLORING. In this protocol, Arthur verifies that an $n$-vertex graph $G$ is 3-colorable, using two unentangled witnesses with only $O(\log n)$ qubits each. There is a crucial caveat, though: if $G$ is not 3-colorable, then Arthur can only detect this with probability $\Omega\left(1 / n^{6}\right)$ rather than constant probability. ${ }^{2}$

\subsection{Our results}

In this paper, we present new results about all three problems listed previously. Our main results are as follows:

Proving 3SAT with $\widetilde{O}(\sqrt{m})$ qubits. In Section 3, we give a protocol by which Arthur can verify that a 3SAT instance of size $m$ has a satisfying assignment, using $O(\sqrt{m}$ polylog $m)$ unentangled witnesses with $O(\log m)$ qubits each. Of course, this is a larger number of qubits than in the protocol of Blier and Tapp [7], but the point is that Arthur can detect cheating with constant probability. Our protocol relies on the PCP Theorem, and in particular, on the existence of PCP's of size $O(m$ polylog $m)$, which was recently shown by Dinur [11].

Weak additivity implies amplification. In Section 4, we reduce several open problems about QMA $(k)$ to weak versions of the Additivity Conjecture in quantum information theory. (In an earlier version of this paper, we pointed out that the weak versions would suffice, but based our results on the original Additivity Conjecture, which was widely believed at the time. Then, as the paper was undergoing final revisions, Hastings [13] announced a spectacular disproof of the Additivity Conjecture.) In particular, we show that weak versions of Additivity Conjecture imply that any QMA(2) protocol can be amplified to exponentially small error, that the "QMA $(k)$ hierarchy" collapses to $\mathrm{QMA}(2)$, and that forcing the Merlins' witnesses to be identical does not change the power of QMA(2).

Nonexistence of perfect disentanglers. In Section 5, we rule out one possible approach to showing QMA $(2)=$ QMA, by proving an extremely simple result that nevertheless seems new and might be of interest. Namely, given finite-dimensional Hilbert spaces $\mathcal{H}, \mathcal{K}$, there is no quantum operation mapping the set of all states in $\mathcal{H}$ to the set of all separable states in $\mathcal{K} \otimes \mathcal{K}$.

Note: In an earlier version of this paper, we claimed to give evidence that QMA $(2) \subseteq$ PSPACE, by showing that this would follow from what we called the "Strong Amplification Conjecture": that it is possible to amplify any QMA(2) protocol, in such a way that one of the Merlin's Hilbert space dimensions remains small compared to the inverse of the error bound. (The trivial upper bound is $\mathrm{QMA}(2) \subseteq \mathrm{NEXP}$, which follows by just guessing exponential-size classical descriptions of the $k$ quantum proofs.) However, Fernando Brandão subsequently pointed out to us that the Strong Amplification

\footnotetext{
${ }^{2}$ Indeed, if the soundness gap were constant rather than $1 /$ poly $(n)$, then Blier and Tapp's protocol could presumably be "scaled up by an exponential" to show QMA $(2)=$ NEXP!
} 


\section{S. Aaronson, S. Beigi, A. Drucker, B. Fefferman, And P. Shor}

Conjecture would actually imply QMA(2) = QMA! 3 Thus, the Strong Amplification Conjecture now seems very unlikely to be true, and while our result was correct, it has been both superseded and effectively nullified in its import. We have not included it in the current version.

In the remainder of this introduction, we give some intuition behind each of these results.

\subsection{Proving 3SAT with $\widetilde{O}(\sqrt{m})$ qubits}

Let $\varphi$ be a 3SAT instance with $n$ variables. Then how long a proof does Merlin need to send Arthur, to convince him that $\varphi$ is satisfiable? (As usual, Merlin is an omniscient prover and Arthur is a skeptical BPP verifier.)

Intuitively, it seems the answer should be about $n$ bits. Certainly, if sublinear-size proofs of satisfiability existed, then $3 \mathrm{~S}$ AT would be in solvable in $2^{o(n)}$ time, since Arthur could just loop over all possible proofs until he found one that worked. Even in the quantum case, one can make a similar statement: if quantum proofs of satisfiability with $o(n)$ qubits existed, then 3 SAT would have a $2^{o(n)}$-time quantum algorithm.

On the other hand, suppose Arthur is given several quantum proofs, which are guaranteed to be unentangled with each other. Then the previous argument seems no longer to work. And this at least raises the possibility that $3 \mathrm{SAT}$ might have sublinear proofs in this setting.

We will show that this possibility is realized:

Theorem 1.1. Let $\varphi$ be a satisfiable $3 \mathrm{SAT}$ instance with $n$ variables and $m \geq n$ clauses. Then one can prove the satisfiability of $\varphi$, with perfect completeness and constant soundness, using $O(\sqrt{m}$ polylog $m)$ unentangled quantum proofs, each with $O(\log m)$ qubits.

In particular, if $m=O(n),{ }^{4}$ then we get an almost-quadratic improvement over the best known witness size in the classical world (or for that matter, in the quantum world with one prover).

Obviously, Theorem 1.1 does not immediately generalize to all NP-complete problems, since the blowup in reducing to $3 \mathrm{~S}$ AT could be more than quadratic. It is an interesting question for which NPcomplete problems an analogue of Theorem 1.1 holds and for which it does not.

\footnotetext{
${ }^{3}$ Here is a sketch of the argument, which we are grateful to Brandão for allowing us to include. If we have two Merlins $A$ and $B$, and the witness of $A$ has only $s(n)$ qubits, then any bipartite pure state $\left|\psi_{A B}\right\rangle$ can be decomposed in Schmidt form as

$$
\left|\psi_{A B}\right\rangle=\sum_{i=1}^{2^{s(n)}} \lambda_{i}\left|\phi_{i}\right\rangle_{A}\left|\varphi_{i}\right\rangle_{B}
$$

where the $\left|\phi_{i}\right\rangle_{A}\left|\varphi_{i}\right\rangle_{B}$ 's are all orthogonal to each other, regardless of the size of $B$. Now, by assumption, every unentangled state of the form $\left|\phi_{i}\right\rangle_{A}\left|\varphi_{i}\right\rangle_{B}$ causes Arthur's amplified protocol to accept with only a tiny probability-without loss of generality, less than $2^{-2 s(n)}$. But this means that Arthur's acceptance probability on $\left|\psi_{A B}\right\rangle$ can be at most

$$
2^{-2 s(n)} \sum_{i=1}^{2^{s(n)}} \lambda_{i}^{*} \lambda_{j} \leq 2^{-2 s(n)}\left(\sum_{i=1}^{2^{s(n)}}\left|\lambda_{i}\right|\right)^{2} \leq 2^{-s(n)}
$$

by Cauchy-Schwarz. Therefore, the amplified QMA(2) protocol is still sound even if the Merlins entangle their witnesses, and hence the language being verified is in QMA.

${ }^{4}$ Note that setting $m=O(n)$ is fairly common in the study of 3S AT, and indeed, the "hardest" random 3SAT instances are believed to occur around $m \approx 4.25 n$.
} 


\section{The Power of Unentanglement}

We now explain the intuition behind Theorem 1.1. The first step in our protocol is to reduce $3 \mathrm{~S}$ AT to a more convenient problem called 2-OUT-OF-4-SAT, where every clause has exactly four literals, and is satisfied if and only if exactly two of the literals are. We also want our 2-OUT-OF-4-SAT instance to be a PCP: that is, either it should be satisfiable, or else at most a $1-\varepsilon$ fraction of clauses should be satisfiable for some constant $\varepsilon>0$. Finally we want the instance to be bounded-literal, meaning that every variable occurs in at most a constant number of clauses. Fortunately, we can get all of this via known classical reductions, including the improved PCP Theorem of Dinur [11], which increase the number of variables and clauses by at most a polylog $m$ factor.

So suppose Arthur has applied these reductions, to obtain a bounded-literal 2-OUT-OF-4-SAT PCP instance $\phi$ with $N$ variables. And now suppose Merlin sends Arthur a $\log N$-qubit quantum state of the form

$$
|\psi\rangle=\frac{1}{\sqrt{N}} \sum_{i=1}^{N}(-1)^{x_{i}}|i\rangle
$$

where $x_{1}, \ldots, x_{N} \in\{0,1\}^{N}$ is the claimed satisfying assignment for $\phi$. (We call a state having the above form a proper state.) Then we show that Arthur can check the veracity of $x_{1}, \ldots, x_{N}$ with perfect completeness and constant soundness. To do so, Arthur simply measures $|\psi\rangle$ in a basis corresponding to the clauses of $\phi$. With constant probability, he will get an outcome of the form

$$
(-1)^{x_{i}}|i\rangle+(-1)^{x_{j}}|j\rangle+(-1)^{x_{k}}|k\rangle+(-1)^{x_{\ell}}|\ell\rangle
$$

where $(i, j, k, \ell)$ is a randomly chosen clause of $\phi$. Assuming this occurs, Arthur can perform a measurement that accepts with certainty if $x_{i}+x_{j}+x_{k}+x_{\ell}=2$ and rejects with constant probability otherwise.

Thus, if only Arthur could somehow assume $|\psi\rangle$ was proper, we would have a $\log N$-qubit witness for 3SAT! The problem, of course, is that Arthur has no way of knowing whether Merlin has cheated and given him an improper state. For example, what if Merlin concentrates the amplitude of $|\psi\rangle$ on some small subset of basis states, and simply omits the other basis states?

Our key technical contribution is to show that, if Arthur gets not one but $O(\sqrt{N})$ copies of $|\psi\rangle$, then he can check with constant soundness whether $|\psi\rangle$ is proper or far from any proper state. Indeed, even if Arthur is given $K=O(\sqrt{N})$ states $\left|\varphi_{1}\right\rangle, \ldots,\left|\varphi_{K}\right\rangle$ which are not necessarily identical, so long as the states are not entangled with each other Arthur can check with constant soundness whether most of them are close to some proper state $|\psi\rangle$. This then yields a protocol for 3SAT with constant soundness and $O(\sqrt{N})$ unentangled proofs of size $O(\log N)$-for Arthur can just choose randomly whether to perform the satisfiability test described above, or to check whether most of the $\left|\varphi_{k}\right\rangle$ 's are close to some proper state $|\psi\rangle$.

To check that most of the states are at least close to each other, Arthur simply has to perform a "swap test" between (say) $\left|\varphi_{1}\right\rangle$ and a random other state $\left|\varphi_{k}\right\rangle$. So the problem is reduced to the following: assuming most of the $\left|\varphi_{k}\right\rangle$ 's are close to $\left|\varphi_{1}\right\rangle$, how can Arthur decide whether $\left|\varphi_{1}\right\rangle$ is proper or far from any proper state?

In our protocol, Arthur does this by first choosing a matching $\mathcal{M}$ on the set $\{1, \ldots, N\}$ uniformly at random. He then measures each state $\left|\varphi_{k}\right\rangle$ in an orthonormal basis that contains the vectors $|i\rangle+|j\rangle$ and $|i\rangle-|j\rangle$ for every edge $(i, j) \in \mathcal{M}$.

Let us think about what happens when Arthur does this. Since he is performing $O(\sqrt{N})$ measurements on almost-identical states, and since each measurement has $N$ possible outcomes, by using a 


\section{S. Aaronson, S. Beigi, A. Drucker, B. Fefferman, And P. Shor}

suitable generalization of the Birthday Paradox one can prove that with $\Omega(1)$ probability, Arthur will find a collision: that is, two outcomes of the form $|i\rangle \pm|j\rangle$, for the same edge $(i, j) \in \mathcal{M}$. So suppose this happens. Then if the $\left|\varphi_{k}\right\rangle$ 's are all equal to a proper state $|\psi\rangle=\sum_{i=1}^{N}(-1)^{x_{i}}|i\rangle$, the two outcomes will clearly "agree": that is, they will either both be $|i\rangle+|j\rangle$ (if $x_{i}=x_{j}$ ) or both be $|i\rangle-|j\rangle$ (if $x_{i} \neq x_{j}$ ). On the other hand, suppose the $\left|\varphi_{k}\right\rangle$ 's are far from any proper state. In that case, we show that the outcomes will "disagree" (that is, one will be $|i\rangle+|j\rangle$ and the other will be $|i\rangle-|j\rangle$ ) with $\Omega(1)$ probability.

To understand why, consider that there are two ways for a state $|\varphi\rangle=\sum_{i=1}^{N} \alpha_{i}|i\rangle$ to be far from proper. First, the probability distribution $\left(\left|\alpha_{1}\right|^{2}, \ldots,\left|\alpha_{N}\right|^{2}\right)$, which corresponds to measuring $|\varphi\rangle$ in the standard basis, could be far from the uniform distribution. Second, the $\alpha_{i}$ 's could be roughly equal in magnitude, but they could have complex phases that cause $|\varphi\rangle$ to be far from any state involving positive and negative real amplitudes only. In either case, though, if Arthur measures according to a random matching $\mathcal{M}$, then with high probability he will obtain an outcome $\alpha_{i}|i\rangle+\alpha_{j}|j\rangle$ that is not close to either $|i\rangle+|j\rangle$ or $|i\rangle-|j\rangle$.

As one would imagine, making all of these claims quantitative and proving them requires a good deal of work.

The reason we need the assumption of unentanglement is that without it, cheating Merlins might correlate their states in such a way that a swap test between any two states passes with certainty, and yet no collisions are ever observed. As we point out in Section 3.7, it seems unlikely that the assumption of unentanglement can be removed, since this would lead to a $2^{\widetilde{O}(\sqrt{m})}$-time classical algorithm for 3SAT. On the other hand, we believe it should be possible to improve our protocol to one involving only two unentangled proofs. This is a problem we leave to future work.

\subsection{Weak additivity implies amplification}

In the one-prover case, it is easy to amplify a QMA protocol with constant error to a protocol with exponentially small error. Merlin simply sends Arthur $m=\operatorname{poly}(n)$ copies of his proof; then Arthur checks each of the copies and outputs the majority answer. To show that this works, the key observation is that Merlin cannot gain anything by entangling the $m$ proofs. Indeed, because of the convexity of Arthur's acceptance probability, Merlin might as well send Arthur an unentangled state $|\psi\rangle^{\otimes m}$, in which case the completeness and soundness errors will decrease exponentially with $m$ by the usual Chernoff bound.

Now suppose we try the same argument for QMA(2). If we ask each Merlin to send $m$ copies of his state, each Merlin might cheat by instead sending an entangled state on $m$ registers. And in that case, as soon as Arthur checks the first copy (consisting of one register from Merlin $_{A}$ and one from Merlin $_{B}$ ), his doing so might create entanglement in the remaining copies where there was none before! This is because of a counterintuitive phenomenon called entanglement swapping [30], by which two quantum systems that have never interacted in the past can nevertheless become entangled, provided those systems are entangled with other systems on which an entangling measurement is performed.

Let us give a small illustration of this phenomenon. Suppose that each "proof" is a single qubit, and that Arthur asks for two proofs from each Merlin (thus, 4 qubits in total). Then if Merlin $_{A}$ is dishonest, he might send Arthur the entangled state $\left|\psi_{A}\right\rangle=|00\rangle+|11\rangle$, and likewise Merlin ${ }_{B} \operatorname{might}$ send Arthur $\left|\psi_{B}\right\rangle=|00\rangle+|11\rangle$ (omitting normalization). Now suppose Arthur measures the qubits $\left|\psi_{A}\right\rangle_{(1)}$ and 


\section{The Power of Unentanglement}

$\left|\psi_{B}\right\rangle_{(1)}$ in the "Bell basis," consisting of the four entangled states $|00\rangle+|11\rangle,|00\rangle-|11\rangle,|01\rangle+|10\rangle$, and $|01\rangle-|10\rangle$. Then conditioned on the outcome of this measurement, it is not hard to see that the joint state of $\left|\psi_{A}\right\rangle_{(2)}$ and $\left|\psi_{B}\right\rangle_{(2)}$ will also be entangled. ${ }^{5}$

Of course, as soon as the remaining $m-1$ copies become entangled, we lose our soundness guarantee and the proof of amplification fails.

Nevertheless, there is a natural amplification procedure that seems like it ought to be robust against such "pathological" behavior. Suppose Arthur chooses the number of copies $m$ to be very large, say $n^{10}$ (much larger than the number of copies he is actually going to check), and suppose that each copy he does check is chosen uniformly at random. Then whatever entanglement Arthur produces during the checking process ought be "spread out" among the copies, so that with high probability, every copy that Arthur actually encounters is close to separable.

It follows, from the "finite quantum de Finetti theorem" of König and Renner [20], that if the number of copies were large enough then the above argument would work. Unfortunately, the number of copies needs to be exponential in $n$ for that theorem to apply.

We will show that the argument works with poly $(n)$ copies, provided one can formalize terms like "spread out" and "close to separable" using a suitable measure of entanglement. The only problem, then, is that a measure of entanglement with the properties we want is not yet known to exist! Informally, we want an entanglement measure $E$ that

(i) is superadditive (meaning it "spreads itself out" among registers), and

(ii) is faithful (meaning if $E(\rho)$ is polynomially small then $\rho$ is polynomially close to a separable state in trace distance).

Among existing entanglement measures, there is one-the entanglement of formation $E_{F}$, introduced by Bennett et al. [6] - that is known to satisfy (ii), and that until recently was conjectured to satisfy (i). ${ }^{6}$ This conjecture is known to be equivalent to the Additivity Conjecture from quantum information theory [27]. Thus, an earlier version of this paper assumed the Additivity Conjecture in proving several results.

In a dramatic recent development, Hastings [13] has shown that the Additivity Conjecture is false. Fortunately, our results require only weak, asymptotic versions of the Additivity Conjecture, which we still conjecture are true, and which are stated formally in Section 4.1.

Our first result says that, if a weak Additivity Conjecture holds, then any QMA(2) protocol can be amplified to exponentially small error. We also prove, unconditionally, that any QMA $(k)$ protocol with constant soundness can be simulated by a QMA(2) protocol with $\Omega(1 / k)$ soundness. Combining these two results, we find that if a weak Additivity Conjecture holds, then $\mathrm{QMA}(k)=\mathrm{QMA}(2)$ for all $k \geq 2 .{ }^{7}$ Another interesting consequence we get is that, assuming a weak Additivity Conjecture, $k$ Merlins who

\footnotetext{
${ }^{5}$ Indeed, this example can be seen as a special case of quantum teleportation [5]: Arthur uses the entanglement between $\operatorname{Merlin}_{A}$ 's left and right registers, as well as between Merlin $_{B}$ 's left and right registers, to teleport an entangled state into the two right registers by acting only on the two left registers.

${ }^{6}$ There is also another measure — the squashed entanglement $E_{s q}$, introduced by Christandl and Winter [10] — that is known to satisfy (i), but unfortunately can be shown not to satisfy (ii).

${ }^{7}$ Kobayashi et al. (personal communication) have shown independently of us that if QMA(2) protocols can be amplified to exponentially small error, then $\mathrm{QMA}(k)=\mathrm{QMA}(2)$ for all $k \geq 2$.
} 
all send copies of the same witness yield the same computational power as $k$ Merlins who can send different witnesses.

\subsection{Nonexistence of perfect disentanglers}

While we now have a few examples where multiple quantum proofs seem to help-such as the 3SAT protocol of this paper, and the pure state $N$-representability problem [21]—we still have no "complexitytheoretic" evidence that QMA $(2) \neq Q M A$. Indeed, even proving an oracle separation between QMA (2) and QMA seems extremely difficult.

Thus, let us consider the other direction and try to prove these classes are the same. Potentially the first approach would be to equip Arthur with a disentangler: that is, a quantum operation that would convert Merlin's (possibly-entangled) witness into a separable witness, and thereby let Arthur simulate a QMA(2) protocol in QMA. In this paper we take a first step in the study of disentanglers, by proving that in finite-dimensional Hilbert spaces, there is no operation that produces all and only the separable states as output.

Note that, if we are willing to settle for there being an output close to every separable state, then a disentangler does exist: simply take as input a classical description of the separable state $\sigma$ to be prepared, measure that description in the computational basis, and then prepare $\sigma{ }^{8}$

Likewise, if we are willing to settle for every output being close to a separable state, then a disentangler also exists. For some sufficiently large $N$, take as input a quantum state on registers $R_{0}, R_{1}, \ldots, R_{N}$, choose an index $i \in[N]$ uniformly at random, and output the joint state of $R_{0}$ and $R_{i}$ (discarding everything else). It follows, from the finite quantum de Finetti theorem [20], that with high probability this state will be close to separable.

The key problem with both of these approaches is that the input Hilbert space needs to be exponentially larger than the output Hilbert space. In the case of the "de Finetti approach," this follows from considering the maximally antisymmetric state

$$
\frac{1}{\sqrt{N !}} \sum_{\sigma \in S_{N}}(-1)^{\operatorname{sgn}(\sigma)}|\sigma(1)\rangle \cdots|\sigma(N)\rangle,
$$

which has the properties that

(i) there are exponentially many registers (as a function of $n=\log N$, the size of a given register), but

(ii) the reduced state of any two registers is far from a separable state.

Watrous (personal communication) has conjectured that this exponentiality is an unavoidable feature of any approximate disentangler. Proving or disproving this remains one of the central open problems about QMA(2).

\footnotetext{
${ }^{8}$ This example also shows that our result fails if the input space is infinite-dimensional-for then one could give an infinitely-precise description of $\sigma$.
} 


\subsection{Table of contents}

The rest of the paper is organized as follows.

Section 2 Preliminaries

Section 3 Proving 3S AT with $\widetilde{O}(\sqrt{m})$ qubits

Section 4 Weak additivity implies amplification (as well as $\mathrm{QMA}(k)=\mathrm{QMA}(2)$, etc.)

Section 5 Nonexistence of perfect disentanglers

Section 6 List of open problems

\section{Preliminaries}

In this section, we first define the complexity class $\mathrm{QMA}(k, a, b)$, or Quantum Merlin-Arthur with $k$ unentangled witnesses and error bounds $a, b$, and state some basic facts and conjectures about this class. We then survey some concepts from quantum information theory we will need, including trace distance, superoperators, and the swap test.

\subsection{Multiple-prover QMA}

Definition 2.1. A language $L$ is in $\operatorname{QMA}(k, a, b)$ if there exists a polynomial-time quantum algorithm $Q$ such that for all inputs $x \in\{0,1\}^{n}$ :

(i) If $x \in L$ then there exist witnesses $\left|\psi_{1}\right\rangle, \ldots,\left|\psi_{k}\right\rangle$, with poly $(n)$ qubits each, such that $Q$ accepts with probability at least $b$ given $|x\rangle \otimes\left|\psi_{1}\right\rangle \otimes \cdots \otimes\left|\psi_{k}\right\rangle$.

(ii) If $x \notin L$ then $Q$ accepts with probability at most $a$ given $|x\rangle \otimes\left|\psi_{1}\right\rangle \otimes \cdots \otimes\left|\psi_{k}\right\rangle$, for all $\left|\psi_{1}\right\rangle, \ldots,\left|\psi_{k}\right\rangle$.

As a convention, we also define $\mathrm{QMA}(k):=\mathrm{QMA}(k, 1 / 3,2 / 3)$, and $\mathrm{QMA}:=\mathrm{QMA}(1) .{ }^{9}$

The above definition makes sense for all integers $k$ from 1 up to $\operatorname{poly}(n)$, and nonnegative real functions $2^{-\operatorname{poly}(n)} \leq a(n)<b(n) \leq 1-2^{-\operatorname{poly}(n)}$.

In the one-prover case, we know that $\mathrm{QMA}(1,1 / 3,2 / 3)=\mathrm{QMA}\left(1,2^{-p(n)}, 1-2^{-p(n)}\right)$ for all polynomials $p$ (see [22] for example). This is what justifies the convention $\mathrm{QMA}(1)=\mathrm{QMA}(1,1 / 3,2 / 3)$. By contrast, we do not yet know whether the convention $\operatorname{QMA}(k)=\mathrm{QMA}(k, 1 / 3,2 / 3)$ is justified for $k \geq 2$. That it $i$ justified is the content of the following conjecture:

Conjecture 2.2 (Amplification). $\mathrm{QMA}(k, a, b)=\mathrm{QMA}\left(k, 2^{-p(n)}, 1-2^{-p(n)}\right)$ for all $k$, all $a<b$ with $b-a=\Omega(1 / \operatorname{poly}(n))$, and all polynomials $p$.

One is tempted to make an even stronger conjecture: that the entire hierarchy of QMA $(k, a, b)$ 's we have defined collapses to just two complexity classes, namely QMA and QMA(2).

\footnotetext{
${ }^{9}$ For purposes of definition, we assume we have fixed a specific machine model (e. g., a universal set of quantum gates) though if the Amplification Conjecture to be discussed shortly holds, then this choice will turn out not to matter.
} 


\section{S. Aaronson, S. Beigi, A. Drucker, B. Fefferman, And P. Shor}

Conjecture 2.3 (Collapse). $\mathrm{QMA}(k, a, b)=\mathrm{QMA}\left(2,2^{-p(n)}, 1-2^{-p(n)}\right)$ for all $k \geq 2$, all $a<b$ with $b-a=\Omega(1 / \operatorname{poly}(n))$, and all polynomials $p$.

The main progress so far on these conjectures has been due to Kobayashi et al. [19], who showed that the Amplification and Collapse Conjectures are actually equivalent:

Theorem 2.4 ([19]). Conjecture 2.2 implies Conjecture 2.3.

Let us observe that one can make the completeness error (though not the soundness error) exponentially small, using a simple argument based on Markov's inequality. We will need this observation in Section 4.

Lemma 2.5. $\mathrm{QMA}(k, a, b) \subseteq \mathrm{QMA}\left(k, 1-(b-a), 1-2^{-p(n)}\right)$ for all $k$, all $a<b<1$, and all polynomials $p$.

Proof. We use the following protocol. Each Merlin provides $m=C \cdot p(n) /(b-a)^{2}$ registers for some constant $C$. Then Arthur runs his verification procedure $m$ times in parallel, once with each $k$-tuple of registers, and accepts if and only if at least a $d$ fraction of invocations accept, for some $d$ slightly less than $b$.

To show completeness, we use a Chernoff bound. Assuming the Merlins are honest, each one simply provides $m$ copies of his witness. Then on each invocation, Arthur accepts with independent probability at least $b$. So assuming we chose a sufficiently large constant $C$, the probability that Arthur accepts less than $d m$ times is at most $2^{-p(n)}$.

To show soundness, we use Markov's inequality. The expected number of accepting invocations is at most am (by linearity of expectation, this is true even if the registers are entangled). Hence the probability that this number exceeds $d m$ is at most $a / d$, which we can ensure is less than $1-(b-a)$ by choosing $d \in\left(\frac{a}{1-(b-a)}, b\right)$ (note that such a $d$ must exist by the assumption $a<b<1$ ).

\subsection{Quantum information}

We now review some quantum information concepts that we will need. For more details see Nielsen and Chuang [23] for example.

Given two mixed states $\rho$ and $\sigma$, their trace distance is $\|\rho-\sigma\|_{\operatorname{tr}}:=\frac{1}{2} \sum_{i=1}^{n}\left|\lambda_{i}\right|$, where $\left(\lambda_{1}, \ldots, \lambda_{n}\right)$ are the eigenvalues of $\rho-\sigma$. We say $\sigma$ is $\varepsilon$-close to $\rho$ if $\|\rho-\sigma\|_{\text {tr }} \leq \varepsilon$, and $\varepsilon$-far otherwise. The importance of trace distance comes from the following fact:

Proposition 2.6. Suppose $\sigma$ is $\varepsilon$-close to $\rho$. Then any measurement that accepts $\rho$ with probability $p$, accepts $\sigma$ with probability at most $p+\varepsilon$.

Trace distance also satisfies the triangle inequality:

Proposition 2.7. $\|\rho-\sigma\|_{\text {tr }}+\|\sigma-\xi\|_{\text {tr }} \geq\|\rho-\xi\|_{\text {tr }}$ for all $\rho, \sigma, \xi$.

Given a pure state $|\psi\rangle$ and a mixed state $\rho$, their squared fidelity $\langle\psi|\rho| \psi\rangle$ is the probability of obtaining $|\psi\rangle$ as the result of a projective measurement on $\rho$. Squared fidelity behaves nicely under tensor products: 
Proposition 2.8. Given a $k$-partite state $\rho^{A_{1} A_{2} \cdots A_{k}}$, suppose there are pure states $\left|\psi_{1}\right\rangle, \ldots,\left|\psi_{k}\right\rangle$ such that $\left\langle\psi_{i}\left|\rho^{A_{i}}\right| \psi_{i}\right\rangle \geq 1-\varepsilon_{i}$ for all $i$. Let $|\Psi\rangle:=\left|\psi_{1}\right\rangle \otimes \cdots \otimes\left|\psi_{k}\right\rangle$ and $\varepsilon:=\varepsilon_{1}+\cdots+\varepsilon_{k}$. Then

$$
\left\langle\Psi\left|\rho^{A_{1} A_{2} \cdots A_{k}}\right| \Psi\right\rangle \geq 1-\varepsilon
$$

Proof. We can assume without loss of generality that $\left|\psi_{i}\right\rangle=|0\rangle$ for all $i$. Then each $\rho^{A_{i}}$, when measured in the standard basis, yields the outcome $|0\rangle$ with probability at least $1-\varepsilon_{i}$. By the union bound, it follows that $\rho^{A_{1} A_{2} \cdots A_{k}}$, when measured in the standard basis, yields the outcome $|\Psi\rangle=|0\rangle^{\otimes k}$ with probability at least $1-\varepsilon$. Hence $\left\langle\Psi\left|\rho^{A_{1} A_{2} \cdots A_{k}}\right| \Psi\right\rangle \geq 1-\varepsilon$.

Trace distance and squared fidelity are related to each other as follows:

Proposition 2.9. For all $\rho$ and $|\psi\rangle$,

$$
\langle\psi|\rho| \psi\rangle+\| \rho-|\psi\rangle\langle\psi| \|_{\text {tr }}^{2} \leq 1
$$

The most general kind of operation on quantum states is called a superoperator. Any superoperator $\Phi$ acting on $n$ qubits can be expressed in the following operator-sum representation:

$$
\Phi(\rho)=\sum_{i=1}^{2 n} E_{i} \rho E_{i}^{\dagger}, \quad \text { where } \quad \sum_{i=1}^{2^{2 n}} E_{i}^{\dagger} E_{i}=I
$$

Given a product state $\rho \otimes \sigma$, the swap test is a quantum operation that measures the overlap between $\rho$ and $\sigma$. The test accepts with probability $\frac{1}{2}(1+\operatorname{tr}(\rho \sigma))$ and rejects otherwise. The swap test can also reveal information about the purity of a state, as follows:

Proposition 2.10. Suppose $\langle\psi|\rho| \psi\rangle<1-\varepsilon$ for all pure states $|\psi\rangle$. Then a swap test between $\rho$ and any other state rejects with probability greater than $\varepsilon / 2$.

Proof. Choose a basis that diagonalizes $\rho$, so that $\rho=\operatorname{diag}\left(\lambda_{1}, \ldots, \lambda_{N}\right)$ where $\lambda_{1}, \ldots, \lambda_{N}$ are $\rho$ 's eigenvalues. By assumption, $\lambda_{i}<1-\varepsilon$ for every $i$. So given any mixed state $\sigma$, a swap test between $\rho$ and $\sigma$ accepts with probability

$$
\frac{1+\operatorname{tr}(\rho \sigma)}{2}=\frac{1}{2}+\frac{1}{2} \sum_{i=1}^{N} \lambda_{i} \sigma_{i i}<\frac{1}{2}+\frac{1-\varepsilon}{2} \sum_{i=1}^{N} \sigma_{i i}=1-\frac{\varepsilon}{2} .
$$

\section{Proving 3SAT with quadratically fewer qubits}

We now present our protocol for proving the satisfiability of a 3S AT instance of size $m$, using $\widetilde{O}(\sqrt{m})$ unentangled quantum proofs with $O(\log m)$ qubits each. For ease of presentation, the protocol will be broken into a sequence of four steps:

(1) In Section 3.1, we give a sequence of classical reductions, from the original 3SAT problem to a different NP-complete problem that we will actually use. 
(2) In Section 3.2, we describe a protocol for the special case where Merlin's message to Arthur is "proper": that is, of the form $\frac{1}{\sqrt{N}} \sum_{i=1}^{N}(-1)^{x_{i}}|i\rangle$ for some Boolean $x_{1}, \ldots, x_{N}$.

(3) In Section 3.3, we generalize our protocol to the case where the Merlins send Arthur $\widetilde{O}(\sqrt{m})$ witnesses, which are not necessarily proper but which are guaranteed to be identical to each other.

(4) In Section 3.5, we remove the restriction that the states be identical.

We end in Section 3.7 with some general observations about our protocol and the prospects for improving it further.

\subsection{Classical reductions}

It will be convenient to work not with $3 \mathrm{SAT}$ but with a related problem called 2-OUT-OF-4-SAT, in which every clause has exactly four literals, and is satisfied if and only if exactly two of the literals are. We will also need our 2-OUT-OF-4-SAT instance to be a PCP, and to be bounded-literal (that is, every variable should appear in at most $O(1)$ clauses). The following lemma shows how to get everything we want with only a polylogarithmic blowup in the number of variables and clauses.

Lemma 3.1. There exists a polynomial-time Karp reduction that maps a 3SAT instance $\varphi$ to a 2-OUTOF-4-SAT instance $\phi$, and that has the following properties:

(i) If $\varphi$ has $n$ variables and $m \geq n$ clauses, then $\phi$ has $O(m$ polylog $m)$ variables and $O(m$ polylog $m)$ clauses.

(ii) Every variable of $\phi$ occurs in at most c clauses, for some constant $c$.

(iii) The reduction is a PCP reduction (meaning that satisfiable instances map to satisfiable instances, while unsatisfiable instances map to instances that are $\varepsilon$-far from satisfiable for some constant $\varepsilon>0)$.

Proof. Given a 3SAT instance $\varphi$, we first amplify its soundness gap to a constant using the celebrated method of Dinur [11]. Next we use a reduction due to Papadimitriou and Yannakakis [25], which makes every variable occur in exactly 29 clauses, without destroying the soundness gap. Finally we use a gadget due to Khanna et al. [14], which converts from 3S AT to 2-OUT-OF-4-SAT, without destroying either the soundness gap or the bounded literal property. Note that the reduction of Dinur [11] incurs only a polylogarithmic blowup in the total size of the instance, while the other two reductions incur a constant blowup.

\subsection{The proper state case}

Suppose Arthur has applied Lemma 3.1, to obtain a bounded-literal 2-OUT-OF-4-SAT instance $\phi$ with $N=O(m$ polylog $m)$ variables, $M=O(m$ polylog $m)$ clauses, and a constant soundness gap $\varepsilon>0$. And now suppose Merlin sends Arthur a $\log N$-qubit state of the form

$$
|\psi\rangle=\frac{1}{\sqrt{N}} \sum_{i=1}^{N}(-1)^{x_{i}}|i\rangle,
$$




\section{The Power of Unentanglement}

where $x_{1}, \ldots, x_{N} \in\{0,1\}^{N}$ is a claimed satisfying assignment for $\phi$. Call a state having the above form (for some Boolean $x_{i}$ 's) a proper state. Then we claim the following:

Lemma 3.2. Assuming $|\psi\rangle$ is proper, Arthur can check whether $\phi$ is satisfiable with perfect completeness and constant soundness.

Proof. To perform the check, Arthur uses the following Satisfiability Test. First he partitions the clauses of $\phi$ into a constant number of blocks $B_{1}, \ldots, B_{s}$, such that within each block, no two clauses share a variable. Such a partition clearly exists by the assumption that $\phi$ is bounded-literal, and furthermore can be found efficiently (e.g., using a greedy algorithm). Next he chooses one of the blocks $B_{r}$ uniformly at random, and measures $|\psi\rangle$ in an orthonormal basis with one projector for each clause in $B_{r}$. Because a single block in the partition of clauses does not necessarily cover all the variables, it is possible that the measurement result will not correspond to any clause in $B_{r}$, in which case Arthur accepts. However, suppose that the measurement yields the following reduced state, for some random clause $C_{i j k \ell}:=(i, j, k, \ell)$ in $B_{r}$ :

$$
\left|\psi_{i j k l}\right\rangle:=\frac{1}{2}\left[(-1)^{x_{i}}|i\rangle+(-1)^{x_{j}}|j\rangle+(-1)^{x_{k}}|k\rangle+(-1)^{x_{\ell}}|\ell\rangle\right] .
$$

Notice that, of the 16 possible assignments to the variables $\left(x_{i}, x_{j}, x_{k}, x_{\ell}\right)$, six of them satisfy $C_{i j k \ell}$, and those six lead to three states $\left|\psi_{i j k \ell}\right\rangle$ that are orthogonal to one another (as well as the negations of those states, which are essentially the same). It follows that Arthur can perform a projective measurement on $\left|\psi_{i j k \ell}\right\rangle$, which accepts with probability 1 if $C_{i j k \ell}$ is satisfied, and rejects with constant probability if $C_{i j k \ell}$ is unsatisfied. Furthermore, because the number of blocks $B_{r}$ is a constant, each of the $M$ clauses of $\phi$ is checked in this test with probability $\Omega(1 / M)$. And we know that, if $x_{1}, \ldots, x_{N}$ is not a satisfying assignment for $\phi$, then a constant fraction of the clauses will be unsatisfied.

Putting everything together, we find that if $\phi$ is satisfiable, then the Satisfiability Test accepts $|\psi\rangle$ with probability 1 ; while if $\phi$ is unsatisfiable, then it rejects with constant probability.

\subsection{The symmetric case}

Thus, the problem we need to solve is "merely" how to force Merlin to send a proper state. For example, how can Arthur prevent a cheating Merlin from concentrating the amplitude of $|\psi\rangle$ on some subset of basis states for which the Satisfiability Test accepts, and omitting the other basis states?

To solve this problem, Arthur is going to need more Merlins. In particular, let us suppose there are $K=\Theta(\sqrt{N})$ unentangled Merlins, who send Arthur $\log N$-qubit states $\left|\varphi_{1}\right\rangle, \ldots,\left|\varphi_{K}\right\rangle$ respectively. By convexity, we can assume without loss of generality that these states are pure. For the time being, we also assume that the states are identical; that is, $\left|\varphi_{i}\right\rangle=|\varphi\rangle$ for all $i \in[K]$. Given these states, Arthur performs one of the following two tests, each with probability $1 / 2$ :

Satisfiability Test: Arthur chooses any copy of $|\varphi\rangle$, and performs the Satisfiability Test described in Section 3.2.

Uniformity Test: Arthur chooses a matching $\mathcal{M}$ on $[N]$ uniformly at random. He then measures each copy of $|\varphi\rangle$ in an orthonormal basis, which contains the vectors

$$
\frac{|i\rangle+|j\rangle}{\sqrt{2}}, \frac{|i\rangle-|j\rangle}{\sqrt{2}}
$$




\section{S. Aaronson, S. Beigi, A. Drucker, B. Fefferman, And P. Shor}

for every edge $(i, j) \in \mathcal{M}$. If for some $(i, j) \in \mathcal{M}$, the two outcomes $\frac{|i\rangle+|j\rangle}{\sqrt{2}}$ and $\frac{|i\rangle-|j\rangle}{\sqrt{2}}$ both occur among the $K$ measurement outcomes, then Arthur rejects. Otherwise he accepts.

It is clear that the above protocol has perfect completeness. For if $\phi$ is satisfiable, then the Merlins can just send $K$ copies of a proper state $|\psi\rangle$ corresponding to a satisfying assignment for $\phi$. In that case, both tests will accept with probability 1 . Our goal is to prove the following:

Theorem 3.3. The protocol has constant soundness (again, assuming the $\left|\varphi_{i}\right\rangle$ 's are all identical).

To prove Theorem 3.3, we need to show that if $\phi$ is unsatisfiable, then one of the two tests rejects with constant probability. There are two cases. First suppose $|\varphi\rangle$ is $\varepsilon$-close in trace distance to some proper state $|\psi\rangle$. Then provided we choose $\varepsilon>0$ sufficiently small, Lemma 3.2, combined with Proposition 2.6, already implies that the Satisfiability Test rejects with constant probability. So our task reduces to proving the following:

Claim 3.4. Suppose $|\varphi\rangle$ is $\varepsilon$-far in trace distance from any proper state $|\psi\rangle$, for some $\varepsilon>0$. Then the Uniformity Test rejects with some constant probability $\delta(\varepsilon)>0$.

In analyzing the Uniformity Test, we say that Arthur finds a collision if he obtains two measurement outcomes of the form $|i\rangle \pm|j\rangle$ for the same $(i, j)$ pair, and that he finds a disagreement if one of the outcomes is $|i\rangle+|j\rangle$ and the other is $|i\rangle-|j\rangle$. Of course, finding a disagreement is what causes him to reject.

The first step, though, is to lower-bound the probability that Arthur finds a collision. Let $|\varphi\rangle=$ $\alpha_{1}|1\rangle+\cdots+\alpha_{N}|N\rangle$. Then for every copy of $|\varphi\rangle$ and every edge $(i, j) \in \mathcal{M}$, Arthur measures an outcome of the form $|i\rangle \pm|j\rangle$ with probability $\left|\alpha_{i}\right|^{2}+\left|\alpha_{j}\right|^{2}$, and these outcomes are independent from one copy to the next. We are interested in the probability that, for some $(i, j)$ pair, Arthur measures $|i\rangle \pm|j\rangle$ more than once. But this is just the famous Birthday Paradox, with $K=\Theta(\sqrt{N})$ "people" (the copies of $|\varphi\rangle$ ) and $N / 2$ "days" (the edges in $\mathcal{M}$ ). The one twist is that the distribution over birthdays need not be uniform. However, a result of Bloom and Knight [8] shows that the Birthday Paradox occurs in the nonuniform case as well:

Lemma 3.5 (Generalized Birthday Paradox [8]). Suppose there are $N$ days in the year, and each person's birthday is drawn independently from the same distribution (not necessarily uniform). Then if there are $\Theta(\sqrt{N})$ people, at least two of them share a birthday with $\Omega(1)$ probability. (Indeed, the probability of a collision is minimized precisely when the distribution over birthdays is uniform.)

Therefore Arthur finds a collision with constant probability. The hard part is to show that he finds a disagreement with constant probability. Here, of course, we will have to use the fact that $|\varphi\rangle$ is $\varepsilon$-far from proper.

For now, let us restrict attention to two copies of $|\varphi\rangle$. For each edge $(i, j) \in \mathcal{M}$, define the "disagreement probability"

$$
p_{i j}=\frac{2\left|\frac{\alpha_{i}+\alpha_{j}}{\sqrt{2}}\right|^{2}\left|\frac{\alpha_{i}-\alpha_{j}}{\sqrt{2}}\right|^{2}}{\left(\left|\alpha_{i}\right|^{2}+\left|\alpha_{j}\right|^{2}\right)^{2}}
$$


to be the probability that, conditioned on measuring two outcomes of the form $|i\rangle \pm|j\rangle$, one of the outcomes is $|i\rangle+|j\rangle$ and the other one is $|i\rangle-|j\rangle$. Also, say an edge $(i, j) \in \mathcal{M}$ is $c$-unbalanced with respect to $|\varphi\rangle$ if $p_{i j} \geq c$, and let $\mathcal{S}_{c} \subseteq \mathcal{M}$ be the set of $c$-unbalanced edges. Say that a set of edges $\mathcal{S} \subseteq \mathcal{M}$ is $d$-large with respect to $|\varphi\rangle$ if

$$
\sum_{(i, j) \in \mathcal{S}}\left(\left|\alpha_{i}\right|^{2}+\left|\alpha_{j}\right|^{2}\right) \geq d
$$

Then the key fact is the following:

Theorem 3.6. Suppose $|\varphi\rangle$ is $\varepsilon$-far in trace distance from any proper state. Then $\mathcal{S}_{c}$ is $d$-large with respect to $|\varphi\rangle$ with probability at least $1 / 3$ over the choice of $\mathcal{M}$, for some constants $c$ and depending on $\varepsilon$.

The proof of Theorem 3.6 is deferred to the next section.

Assuming Theorem 3.6, we can complete the proof of Claim 3.4, and hence of Theorem 3.3. The idea is this: when Arthur performs the Uniformity Test, simply discard all measurement outcomes that are not of the form $|i\rangle \pm|j\rangle$ for some $(i, j) \in \mathcal{S}_{c}$. Assuming $\mathcal{S}_{c}$ is $d$-large-which it is with constant probability by Theorem 3.6-with overwhelming probability that still leaves $\Theta(\sqrt{N})$ "good" measurement outcomes. Then by Lemma 3.5, with constant probability there will be a collision among these good outcomes. And by the definition of $\mathcal{S}_{c}$, any such collision will also be a disagreement with constant probability, thereby causing Arthur to reject.

\subsection{Unbalanced edges in random matchings}

The goal of this section is to prove Theorem 3.6, which we now restate in a more careful way.

Theorem. There exist constants $c, d>0$ for which the following holds. Let $N$ be even and sufficiently large. Suppose the state $|\varphi\rangle=\alpha_{1}|1\rangle+\cdots+\alpha_{N}|N\rangle$ is $\varepsilon$-far in trace distance from any proper state (that is, any state of the form

$$
\frac{1}{\sqrt{N}} \sum_{i=1}^{N}(-1)^{x_{i}}|i\rangle
$$

where $\left.x_{1}, \ldots, x_{N} \in\{0,1\}\right)$. Let $\mathcal{M}$ be a matching on $[N]$ chosen uniformly at random, and let $\mathcal{S}$ be the set of edges $(i, j) \in \mathcal{M}$ that are "c $\varepsilon^{8}$-unbalanced," meaning that

$$
\left|\alpha_{i}^{2}-\alpha_{j}^{2}\right|^{2} \geq 2 c \varepsilon^{8}\left(\left|\alpha_{i}\right|^{2}+\left|\alpha_{j}\right|^{2}\right)^{2}
$$

Then

$$
\sum_{(i, j) \in \mathcal{S}}\left(\left|\alpha_{i}\right|^{2}+\left|\alpha_{j}\right|^{2}\right) \geq d \varepsilon^{4}
$$

with probability at least $1 / 3$ over the choice of $\mathcal{M}$.

Given a state $|\varphi\rangle=\alpha_{1}|1\rangle+\cdots+\alpha_{N}|N\rangle$, define the nonuniformity of $|\varphi\rangle$ to be

$$
\mathrm{NU}(|\varphi\rangle):=\left.\frac{1}{2} \sum_{i=1}^{N}|| \alpha_{i}\right|^{2}-\frac{1}{N} \mid .
$$




\section{S. Aaronson, S. Beigi, A. Drucker, B. Fefferman, And P. Shor}

Intuitively, $\mathrm{NU}(|\varphi\rangle)$ measures whether the distribution induced by measuring $|\varphi\rangle$ in the standard basis is close to uniform or not. We will divide the proof of Theorem 3.6 into two cases: first that $\mathrm{NU}(|\varphi\rangle)>$ $\varepsilon^{4} / 100$ (the "nonuniform case"), and second that $\mathrm{NU}(|\varphi\rangle) \leq \varepsilon^{4} / 100$ (the "uniform case").

\subsubsection{The nonuniform case}

We now prove Theorem 3.6 in the case $\mathrm{NU}(|\varphi\rangle)>\varepsilon^{4} / 100$. For convenience, define $\kappa:=\varepsilon^{4} / 100$ and $p_{i}:=\left|\alpha_{i}\right|^{2}$. Then the condition

$$
\left|\alpha_{i}^{2}-\alpha_{j}^{2}\right|^{2} \geq 2 c \varepsilon^{8}\left(\left|\alpha_{i}\right|^{2}+\left|\alpha_{j}\right|^{2}\right)^{2}
$$

is equivalent to

$$
p_{i}^{2}+p_{j}^{2}-2 \operatorname{Re} \alpha_{i}^{2}{\overline{\alpha_{j}}}^{2} \geq 2 c \varepsilon^{8}\left(p_{i}+p_{j}\right)^{2},
$$

which will certainly be true whenever $\left(p_{i}-p_{j}\right)^{2} \geq 2 c \varepsilon^{8}\left(p_{i}+p_{j}\right)^{2}$, or equivalently

$$
\frac{p_{i}}{p_{j}}+\frac{p_{j}}{p_{i}} \geq \frac{2+2 c \varepsilon^{8}}{1-2 c \varepsilon^{8}}
$$

(If $p_{i}=0$ or $p_{j}=0$ then we stipulate that the above inequality holds.) Thus, it suffices to prove the following classical lemma.

Lemma 3.7. Let $N$ be even and sufficiently large. Let $\left(p_{1}, \ldots, p_{N}\right)$ be a probability distribution, and suppose

$$
\frac{1}{2} \sum_{i=1}^{N}\left|p_{i}-\frac{1}{N}\right| \geq \kappa .
$$

Let $\mathcal{M}$ be a uniform random matching on $[N]$, and let $\mathcal{S}$ be the set of edges $(i, j) \in \mathcal{M}$ such that $p_{i} / p_{j}+$ $p_{j} / p_{i} \geq 2+\kappa^{2} / 16$. Then

$$
\sum_{(i, j) \in \mathcal{S}}\left(p_{i}+p_{j}\right) \geq \frac{\kappa}{12}
$$

with probability at least $1 / 3$ over $\mathcal{M}$.

Let $H$ (the "heavy elements") be the set of $i \in[N]$ such that $p_{i} \geq 1 / N$, and let $H^{*} \subseteq H$ (the "very heavy elements") be the set of $i \in[N]$ such that $p_{i} \geq(1+\kappa / 2) / N$. Let $L$ (the "light elements") be the set of $i \in[N]$ such that $p_{i}<1 / N$, and let $L^{*} \subseteq L$ (the "very light elements") be the set of $i \in[N]$ such that $p_{i} \leq(1-\kappa / 4) / N$. Clearly

$$
\sum_{i \in H}\left(p_{i}-\frac{1}{N}\right)=\sum_{i \in L}\left(\frac{1}{N}-p_{i}\right)=\kappa .
$$

Using this, we can prove two simple facts: that there are $\Omega(N)$ very light elements, and that the very heavy elements have total weight $\Omega(\kappa)$.

Proposition 3.8. $\left|L^{*}\right| \geq \kappa N / 2$. 
Proof. We have

$$
\kappa=\sum_{i \in L}\left(\frac{1}{N}-p_{i}\right) \leq \frac{\left|L^{*}\right|}{N}+\left(|L|-\left|L^{*}\right|\right) \frac{\kappa}{4 N}
$$

Now use $|L| \leq N$ and rearrange.

Given any subset $A \subseteq[N]$, define the "weight" of $A$ to be $W_{A}:=\sum_{i \in A} p_{i}$.

Proposition 3.9. $W_{H^{*}} \geq \kappa / 2$.

Proof. We have

$$
\kappa=\sum_{i \in H}\left(p_{i}-\frac{1}{N}\right)=\sum_{i \in H \backslash H^{*}}\left(p_{i}-\frac{1}{N}\right)+\sum_{i \in H^{*}}\left(p_{i}-\frac{1}{N}\right) \leq N \frac{\kappa}{2 N}+W_{H^{*}} .
$$

Now subtract $\kappa / 2$ from both sides.

To prove Lemma 3.7, we divide into two cases.

The first case is that $|H| \geq N / 2$ (in other words, at least half the elements are heavy). In this case, we begin constructing the matching $\mathcal{M}$ by randomly assigning partners to the "very light elements" $i \in L^{*}$. Recall from Proposition 3.8 that $\left|L^{*}\right| \geq \kappa N / 2$. So by a standard Chernoff bound, it is easy to see that at least (say) $\left|L^{*}\right| / 6$ elements $i \in L^{*}$ will be matched to partners $j \in H$, with probability $1-o(1)$ over $\mathcal{M}$. Notice that every edge $(i, j)$ with $i \in L^{*}$ and $j \in H$ satisfies $p_{i} \leq(1-\kappa / 4) / N$ and $p_{j} \geq 1 / N$, and therefore

$$
\frac{p_{i}}{p_{j}}+\frac{p_{j}}{p_{i}} \geq 1-\kappa / 4+\frac{1}{1-\kappa / 4}>2+\frac{\kappa^{2}}{16} .
$$

Thus, all of these edges go into the set $\mathcal{S}$. We then have

$$
\sum_{(i, j) \in \mathcal{S}}\left(p_{i}+p_{j}\right) \geq \frac{\left|L^{*}\right|}{6} \cdot \frac{1}{N} \geq \frac{\kappa}{12}
$$

and are done.

The second case is that $|H|<N / 2$ (in other words, there are more light elements than heavy ones). In this case, we begin constructing $\mathcal{M}$ by randomly assigning partners to the "very heavy elements" $i \in H^{*}$. Let $B$ be the set of elements $i \in H^{*}$ that get matched to partners in $H$. Then since $|H|<N / 2$, every element of $H^{*}$ goes into $B$ with probability less than $1 / 2$, and hence

$$
\underset{\mathcal{M}}{\mathrm{E}}\left[W_{B}\right]<\sum_{i \in H^{*}} \frac{p_{i}}{2}=\frac{W_{H^{*}}}{2} .
$$

Therefore

$$
\underset{\mathcal{M}}{\operatorname{Pr}}\left[W_{B}>\frac{3}{4} W_{H^{*}}\right]<\frac{2}{3}
$$

by Markov's inequality. In other words, with probability greater than $1 / 3$, at least $1 / 4$ of the probability weight in $H^{*}$ gets matched to partners in $L$. Suppose this happens. 


\section{S. Aaronson, S. Beigi, A. Drucker, B. Fefferman, And P. Shor}

Notice that every edge $(i, j)$ with $i \in H^{*}$ and $j \in L$ satisfies $p_{i} \geq \frac{1+\kappa / 2}{N}$ and $p_{j}<1 / N$, and therefore

$$
\frac{p_{i}}{p_{j}}+\frac{p_{j}}{p_{i}}>1+\kappa / 2+\frac{1}{1+\kappa / 2}>2+\frac{\kappa^{2}}{8}
$$

Thus, all of these edges go into the set $\mathcal{S}$. Furthermore, by the assumption $W_{B} \leq \frac{3}{4} W_{H^{*}}$, we have

$$
\sum_{(i, j) \in \mathcal{S}}\left(p_{i}+p_{j}\right) \geq \sum_{i \in H^{*} \backslash B} p_{i}=W_{H^{*} \backslash B} \geq \frac{W_{H^{*}}}{4} \geq \frac{\kappa}{8}
$$

and are done.

\subsubsection{The uniform case}

We now prove Theorem 3.6 for states $|\varphi\rangle=\alpha_{1}|1\rangle+\cdots+\alpha_{N}|N\rangle$ such that $\mathrm{NU}(|\varphi\rangle) \leq \varepsilon^{4} / 100$. The first step is to define a measure of the distance from $|\varphi\rangle$ to the closest proper state, which we call the impropriety of $|\varphi\rangle$ or $\operatorname{imp}(|\varphi\rangle)$ :

$$
\operatorname{imp}(|\varphi\rangle):=\min _{|r|=1 / N} \sum_{i=1}^{N}\left|\alpha_{i}^{2}-r\right| .
$$

Clearly $0 \leq \operatorname{imp}(|\varphi\rangle) \leq 2$ for all $|\varphi\rangle$, with $\operatorname{imp}(|\varphi\rangle)=0$ if and only if $|\varphi\rangle$ is equivalent to a proper state up to a phase shift. We also have the following:

Lemma 3.10. Suppose $|\varphi\rangle$ is $\varepsilon$-far in trace distance from any proper state. Then $\operatorname{imp}(|\varphi\rangle)>\varepsilon^{2}$.

Proof. By Proposition 2.9, we have $|\langle\varphi \mid \psi\rangle|<\sqrt{1-\varepsilon^{2}}<1-\varepsilon^{2} / 2$ for all proper states $|\psi\rangle$. On the other hand, suppose $\operatorname{imp}(|\varphi\rangle) \leq \varepsilon^{2}$. Then we will construct a proper state $|\psi\rangle$ such that $|\langle\varphi \mid \psi\rangle| \geq 1-\varepsilon^{2} / 2$, thereby obtaining the desired contradiction.

Let $r$ be a complex number with $|r|=1 / N$ that minimizes $\sum_{i=1}^{N}\left|\alpha_{i}^{2}-r\right|$, and let $\sqrt{r}$ be a canonical square root of $r$. Also let $\beta_{i}:=\left|\alpha_{i}^{2}-r\right|$. Then

$$
\left|\alpha_{i}+\sqrt{r}\right|\left|\alpha_{i}-\sqrt{r}\right|=\left|\alpha_{i}^{2}-r\right|=\beta_{i},
$$

which means that either $\left|\alpha_{i}+\sqrt{r}\right| \leq \sqrt{\beta_{i}}$ or $\left|\alpha_{i}-\sqrt{r}\right| \leq \sqrt{\beta_{i}}$. So by setting the $\gamma_{i}$ 's to $\sqrt{r}$ or $-\sqrt{r}$ appropriately, we can construct a state $|\psi\rangle=\gamma_{1}|1\rangle+\cdots+\gamma_{N}|N\rangle$ that is proper up to a trivial phase factor, such that $\left|\alpha_{i}-\gamma_{i}\right| \leq \sqrt{\beta_{i}}$ for all $i$. Then

$$
\begin{aligned}
2-2|\langle\varphi \mid \psi\rangle| & =2-2\left|\sum_{i=1}^{N} \alpha_{i} \overline{\gamma_{i}}\right| \leq 2-\left|\sum_{i=1}^{N} \alpha_{i} \overline{\gamma_{i}}+\sum_{i=1}^{N} \overline{\alpha_{i}} \gamma_{i}\right| \\
& \leq\left|2-\sum_{i=1}^{N} \alpha_{i} \overline{\gamma_{i}}-\sum_{i=1}^{N} \overline{\alpha_{i}} \gamma_{i}\right|=\left|\sum_{i=1}^{N}\left(\left|\alpha_{i}\right|^{2}+\left|\gamma_{i}\right|^{2}-\alpha_{i} \overline{\gamma_{i}}-\overline{\alpha_{i}} \gamma_{i}\right)\right| \\
& =\sum_{i=1}^{N}\left|\alpha_{i}-\gamma_{i}\right|^{2} \leq \sum_{i=1}^{N} \beta_{i}=\operatorname{imp}(|\varphi\rangle) \leq \varepsilon^{2}
\end{aligned}
$$

and hence $|\langle\varphi \mid \psi\rangle| \geq 1-\varepsilon^{2} / 2$ as claimed. 
In what follows, assume $\operatorname{imp}(|\varphi\rangle)>\varepsilon^{2}$.

Now as in Section 3.4.1, let $p_{i}:=\left|\alpha_{i}\right|^{2}$, and for any subset $A \subseteq[N]$, define the "probability weight" of $A$ to be $W_{A}:=\sum_{i \in A} p_{i}$. Also, let $\delta:=\varepsilon^{2} / 5$, and let $U$ (the " $\delta$-uniform subset") be the set of all $i \in[N]$ such that $\left|p_{i}-1 / N\right| \leq \delta / N$. The following proposition shows that $U$ encompasses "most" of $|\varphi\rangle$, whether in terms of cardinality or in terms of probability weight.

Proposition 3.11. $|U| \geq N\left(1-\varepsilon^{2} / 10\right)$ and $W_{U} \geq 1-3 \varepsilon^{2} / 10$.

Proof. We have

$$
\frac{\varepsilon^{4}}{100} \geq \mathrm{NU}(|\varphi\rangle) \geq \frac{1}{2}(N-|U|) \frac{\delta}{N}
$$

hence

$$
|U| \geq N\left(1-\frac{\varepsilon^{4}}{50 \delta}\right)=N\left(1-\frac{\varepsilon^{2}}{10}\right)
$$

hence

$$
W_{U} \geq N\left(1-\frac{\varepsilon^{2}}{10}\right)\left(\frac{1}{N}-\frac{\delta}{N}\right) \geq 1-\frac{3 \varepsilon^{2}}{10}
$$

Let

$$
\operatorname{imp}_{U}(|\varphi\rangle):=\min _{|r|=1 / N} \sum_{i \in U}\left|\alpha_{i}^{2}-r\right|
$$

be an analogue of impropriety that is restricted to the set $U$. By combining Lemma 3.10 with Proposition 3.11, we can now lower-bound $\operatorname{imp}_{U}(|\varphi\rangle)$.

Proposition 3.12. $\operatorname{imp}_{U}(|\varphi\rangle) \geq 3 \varepsilon^{2} / 5$.

Proof. For all $r$ with $|r|=1 / N$, we have

$$
\sum_{i \notin U}\left|\alpha_{i}^{2}-r\right| \leq \sum_{i \notin U} p_{i}+\sum_{i \notin U} \frac{1}{N} \leq\left(1-W_{U}\right)+\frac{N-|U|}{N} \leq \frac{3 \varepsilon^{2}}{10}+\frac{\varepsilon^{2}}{10}=\frac{2 \varepsilon^{2}}{5}
$$

by Proposition 3.11. Hence

$$
\begin{aligned}
\operatorname{imp}_{U}(|\varphi\rangle) & =\min _{|r|=1 / N} \sum_{i \in U}\left|\alpha_{i}^{2}-r\right| \\
& \geq \min _{|r|=1 / N} \sum_{i \in[N]}\left|\alpha_{i}^{2}-r\right|-\max _{|r|=1 / N} \sum_{i \notin U}\left|\alpha_{i}^{2}-r\right| \\
& \geq \operatorname{imp}(|\varphi\rangle)-\frac{2 \varepsilon^{2}}{5} \geq \frac{3 \varepsilon^{2}}{5} .
\end{aligned}
$$




\section{S. Aaronson, S. Beigi, A. Drucker, B. Fefferman, And P. Shor}

We are finally ready for the geometric core of our result. Let $V$ be a collection of vectors in $\mathbb{R}^{2}$ (possibly with multiplicity), which consists of the vector $\left(N \operatorname{Re} \alpha_{i}^{2}, N \operatorname{Im} \alpha_{i}^{2}\right)$ for every $i \in U$. Let $\|v\|$ be the 2-norm of $v$. Then we know by the definition of $U$ that $1-\delta \leq\|v\| \leq 1+\delta$ for all $v \in V$. We also know from Proposition 3.11 that

$$
|V|=|U| \geq N\left(1-\frac{\varepsilon^{2}}{10}\right) \geq 0.9 N
$$

and from Proposition 3.12 that for all unit vectors $w \in \mathbb{R}^{2}$,

$$
\sum_{v \in V}\|v-w\| \geq \frac{3 \varepsilon^{2} N}{5} \geq \frac{3 \varepsilon^{2}|V|}{5} .
$$

Based on this information, we want to find two subsets $X, Y \subseteq V$, both of size $\Omega(|V|)$, such that $\|x-y\|=$ $\Omega(1)$ for all $x \in X$ and $y \in Y$.

For suppose we can do this. Then just as in Section 3.4.1, when a matching $\mathcal{M}$ on $[N]$ is chosen uniformly at random, by a Chernoff bound it will have $\Omega(N)$ edges between the subsets of $[N]$ corresponding to $X$ and $Y$ with overwhelming probability. Assuming that happens, we will have $\left|\alpha_{i}^{2}-\alpha_{j}^{2}\right|=\Omega(1 / N)$ for every such edge $(i, j) \in \mathcal{M}$, and hence all of these edges will get added to the set $\mathcal{S}$. We will therefore have

$$
\sum_{(i, j) \in \mathcal{S}}\left(p_{i}+p_{j}\right)=\Omega(1)
$$

as desired. (For simplicity, we have suppressed the dependence on $\varepsilon$ here.)

What we need, then, is the following geometric lemma.

Lemma 3.13. Let $V$ be a collection of vectors in the plane. Suppose that $1-\delta \leq\|v\| \leq 1+\delta$ for every $v \in V$, and that $\sum_{v \in V}\|v-w\| \geq \kappa|V|$ for every unit vector $w \in \mathbb{R}^{2}$. Then provided $\delta \leq \kappa / 2$, there exist subsets $X, Y \subseteq V$, both of size at least $\kappa|V| / 40$, such that $\|x-y\| \geq \kappa / 20$ for all $x \in X$ and $y \in Y .{ }^{10}$

Proof. Divide the plane into $K \in[30 / \kappa, 40 / \kappa]$ equal-sized, half-open angular sectors, centered about the origin. By the pigeonhole principle, one of these sectors (call it $S$ ) must contain at least $|V| / K \geq \kappa|V| / 40$ of the vectors. Let $S^{\prime}$ be the union of $S$ and its two adjacent sectors. Then we claim that at least $\kappa|V| / 40$ of the vectors must lie outside of $S^{\prime}$. For suppose not. Then let $z$ be the unit vector that bisects $S$, and let

$$
\theta=\frac{3}{2}\left(\frac{2 \pi}{K}\right) \leq \frac{3}{2}\left(\frac{2 \pi}{30 / \kappa}\right)=\frac{\pi \kappa}{10}
$$

be the angle between $z$ and the border of $S^{\prime}$. Notice that by the triangle inequality, we have

$$
\|v-z\| \leq \sqrt{2-2 \cos \theta}+\delta \leq \theta+\delta \leq \frac{\pi \kappa}{10}+\delta
$$

\footnotetext{
${ }^{10}$ We did not try to optimize the constants.
} 
for every $v$ in $S^{\prime}$ (where we have used the bound $\cos \theta \geq 1-\theta^{2} / 2$ ). We also have $\|v-z\| \leq 2+\delta$ for every $v \in V$. Hence

$$
\begin{aligned}
\sum_{v \in V}\|v-z\| & \leq \sum_{v \in S^{\prime}}\left(\frac{\pi \kappa}{10}+\delta\right)+\sum_{v \notin S^{\prime}}(2+\delta) \\
& \leq \frac{\pi \kappa}{10}|V|+2 \frac{\kappa|V|}{40}+\delta|V|<\kappa|V|
\end{aligned}
$$

which is a contradiction.

Now let $X$ be the set of all $v$ 's in $S$, and let $Y$ be the set of all $v$ 's outside $S^{\prime}$. Then $|X| \geq \kappa|V| / 40$ and $|Y| \geq \kappa|V| / 40$. Also, let

$$
\tau=\frac{2 \pi}{K} \geq \frac{\pi \kappa}{20}
$$

be the angle of a single sector. Then it is not hard to see that for all $x \in X$ and $y \in Y$,

$$
\|x-y\| \geq(1-\delta) \sqrt{2-2 \cos \tau} \geq(1-\delta) \frac{\tau}{\sqrt{2}} \geq\left(1-\frac{\kappa}{2}\right) \frac{\pi \kappa}{20 \sqrt{2}} \geq \frac{\kappa}{20}
$$

where we have used the bound $\cos \tau \leq 1-\tau^{2} / 4$ for all $\tau \in[0, \pi / 2]$.

Now set $\kappa:=3 \varepsilon^{2} / 5$. Then $\delta=\varepsilon^{2} / 5<\kappa / 2$ and the condition of Lemma 3.13 is satisfied. So considering the sets $X, Y$ from the lemma, we have

$$
|X|,|Y| \geq \frac{\kappa|V|}{40} \geq \frac{0.9 \kappa N}{40}=\frac{(0.9) 3 \varepsilon^{2} N}{200}>\frac{\varepsilon^{2} N}{100},
$$

and also

$$
\|x-y\| \geq \frac{\kappa}{20} \geq \frac{3 \varepsilon^{2}}{100}
$$

for all $x \in X$ and $y \in Y$. This means that we can find subsets $X^{\prime}, Y^{\prime} \subseteq[N]$ such that

(i) $\left|X^{\prime}\right|,\left|Y^{\prime}\right| \geq \varepsilon^{2} N / 100$ and

(ii) $\left|\alpha_{i}^{2}-\alpha_{j}^{2}\right| \geq \frac{3 \varepsilon^{2}}{100 N}$ for all $i \in X^{\prime}$ and $j \in Y^{\prime}$.

Property (ii) implies that

$$
\left|\alpha_{i}^{2}-\alpha_{j}^{2}\right|^{2} \geq \frac{9 \varepsilon^{4}}{10000 N^{2}} \geq 2 c \varepsilon^{8}\left(\left|\alpha_{i}\right|^{2}+\left|\alpha_{j}\right|^{2}\right)^{2}
$$

for some suitable constant $c$. Hence every edge $(i, j) \in \mathcal{M}$ with $i \in X^{\prime}$ and $j \in Y^{\prime}$ will get added to the set $\mathcal{S}$ (again assuming a suitable $c$ ).

Property (i), together with a Chernoff bound, implies that with probability $1-o(1)$ over the choice of matching $\mathcal{M}$, there are at least (say) $\varepsilon^{4} N / 20000$ edges $(i, j) \in \mathcal{M}$ such that $i \in X^{\prime}$ and $j \in Y^{\prime}$. Suppose this happens. Then

$$
\sum_{(i, j) \in \mathcal{S}}\left(p_{i}+p_{j}\right) \geq \frac{\varepsilon^{4} N}{20000} \cdot 2\left(\frac{1-\delta}{N}\right)=\Omega\left(\varepsilon^{4}\right)
$$

as desired. This completes the proof of Theorem 3.6. 


\subsection{The general case}

Of course, in general the states $\left|\varphi_{1}\right\rangle, \ldots,\left|\varphi_{K}\right\rangle$ sent by the $K=\Theta(\sqrt{N})$ Merlins need not be identical. To deal with this, we now give our final protocol, which removes the symmetry restriction.

\section{The 2-OUT-OF-4-SAT Protocol}

Given $\left|\varphi_{1}\right\rangle, \ldots,\left|\varphi_{K}\right\rangle$, Arthur performs one of the following three tests, each with probability $1 / 3$.

Satisfiability Test: Arthur applies the Satisfiability Test, described in Section 3.2, to $\left|\varphi_{1}\right\rangle$.

Symmetry Test: Arthur chooses an index $k \in\{2, \ldots, K\}$ uniformly at random, performs a swap test between $\left|\varphi_{1}\right\rangle$ and $\left|\varphi_{k}\right\rangle$, and accepts if and only if the swap test accepts.

Uniformity Test: Arthur chooses a matching $\mathcal{M}$ on $[N]$ uniformly at random. He then measures each $\left|\varphi_{k}\right\rangle$ in an orthonormal basis, which contains the vectors

$$
\frac{|i\rangle+|j\rangle}{\sqrt{2}}, \frac{|i\rangle-|j\rangle}{\sqrt{2}}
$$

for every edge $(i, j) \in \mathcal{M}$. If for some $(i, j) \in \mathcal{M}$, the two outcomes $\frac{|i\rangle+|j\rangle}{\sqrt{2}}$ and $\frac{|i\rangle-|j\rangle}{\sqrt{2}}$ both occur among the $K$ measurement outcomes, then Arthur rejects. Otherwise he accepts.

It is clear that the above protocol has perfect completeness, and thus the problem is to show soundness: that is, if $\phi$ is unsatisfiable, then one of the three tests rejects with constant probability. There are three cases.

The first case is that $\left|\varphi_{1}\right\rangle$ is $\varepsilon$-close to some proper state $|\psi\rangle$. Then as before, the Satisfiability Test will reject with constant probability, provided we choose $\varepsilon$ sufficiently small.

The second case is that $\left|\left\langle\varphi_{1} \mid \varphi_{k}\right\rangle\right|<1-\delta$ for at least a $\gamma$ fraction of indices $k \in\{2, \ldots, K\}$. In that case it is clear that the Symmetry Test will reject with probability at least $\gamma \delta / 2$.

The third case is that $\left|\left\langle\varphi_{1} \mid \varphi_{k}\right\rangle\right| \geq 1-\delta$ for more than a $1-\gamma$ fraction of indices $k \in\{2, \ldots, K\}$, but nevertheless $\left|\varphi_{1}\right\rangle$ is $\varepsilon$-far from any proper state. In this case we need to generalize the results of the previous section, to show that the Uniformity Test will still reject with constant probability (dependent on $\varepsilon, \delta$, and $\gamma$ ).

The first step in the analysis is simply to discard all states $\left|\varphi_{k}\right\rangle$ such that $\left|\left\langle\varphi_{1} \mid \varphi_{k}\right\rangle\right|<1-\delta$. By Proposition 2.9, the remaining $K^{\prime} \geq(1-\gamma) K$ states are all $\sqrt{2 \delta}$-close to $\left|\varphi_{1}\right\rangle$ in trace distance.

Now given a matching $\mathcal{M}$ on $[N]$, let $\mathcal{S}_{c}$ be the set of edges in $\mathcal{M}$ that are $c$-unbalanced with respect to $\left|\varphi_{1}\right\rangle$, in the sense defined in Section 3.3. Then Theorem 3.6 implies that $\mathcal{S}_{c}$ is $d$-large with respect to $\left|\varphi_{1}\right\rangle$ (for some constants $c$ and $d$ ) with probability at least $1 / 3$ over the choice of $\mathcal{M}$. Suppose that it is.

Call a measurement outcome $|i\rangle \pm|j\rangle$ good if $(i, j) \in \mathcal{S}_{c}$. Then when Arthur performs the Uniformity Test, we simply discard all states for which the outcome is not good. Since all of the states are $\sqrt{2 \delta}$ close to $\left|\varphi_{1}\right\rangle$, and since $\mathcal{S}_{c}$ is $d$-large with respect to $\left|\varphi_{1}\right\rangle$, with overwhelming probability this still leaves us with $K^{\prime \prime} \approx(d-\sqrt{2 \delta}) K^{\prime}$ states. Call those states $\left|\xi_{1}\right\rangle, \ldots,\left|\xi_{K^{\prime \prime}}\right\rangle$.

Let $\widetilde{\mathcal{M}}=\{|i\rangle \pm|j\rangle:(i, j) \in \mathcal{M}\}$. Given a state $|\varphi\rangle$, let $\mathcal{D}_{|\varphi\rangle}$ be the probability distribution over $\tilde{\mathcal{M}}$ induced by measuring $|\varphi\rangle$ according to $\mathcal{M}$. Then we know that $\left\|\mathcal{D}_{\left|\varphi_{1}\right\rangle}-\mathcal{D}_{\left|\xi_{k}\right\rangle}\right\| \leq \sqrt{2 \delta}$ for all $k \in\left[K^{\prime \prime}\right]$. 


\section{The Power of Unentanglement}

Next let $\mathcal{D}_{|\varphi\rangle}^{\prime}$ be the distribution over $\widetilde{\mathcal{M}}$ induced by measuring $|\varphi\rangle$, and then conditioning on the outcome being good. Then we claim that

$$
\left\|\mathcal{D}_{\left|\xi_{k}\right\rangle}^{\prime}-\mathcal{D}_{\left|\varphi_{1}\right\rangle}^{\prime}\right\| \leq \frac{\sqrt{2 \delta}}{d-\sqrt{2 \delta}}
$$

for all $k \in\left[K^{\prime \prime}\right]$, where as always $\|\cdot\|$ denotes the variation distance. This is so because of the following simple fact:

Proposition 3.14. Let $\mathcal{D}_{1}$ and $\mathcal{D}_{2}$ be probability distributions, let $E$ be an event, and let $\mathcal{D}_{1}^{\prime}$ and $\mathcal{D}_{2}^{\prime}$ denote $\mathcal{D}_{1}$ and $\mathcal{D}_{2}$ respectively conditioned on E. Suppose $\left\|\mathcal{D}_{1}-\mathcal{D}_{2}\right\| \leq \kappa$ and $\operatorname{Pr}_{x \in \mathcal{D}_{1}}[E(x)] \geq a$. Then $\left\|\mathcal{D}_{1}^{\prime}-\mathcal{D}_{2}^{\prime}\right\| \leq \frac{\kappa}{a-\kappa}$.

Proof. Let $b=\operatorname{Pr}_{x \in \mathcal{D}_{2}}[E(x)]$, and note that $|a-b| \leq \kappa$. Also let $p_{x}=\operatorname{Pr}_{\mathcal{D}_{1}}[x]$ and $q_{x}=\operatorname{Pr}_{\mathcal{D}_{2}}[x]$. Then

$$
\begin{aligned}
\left\|\mathcal{D}_{1}^{\prime}-\mathcal{D}_{2}^{\prime}\right\| & =\frac{1}{2} \sum_{x: E(x)}\left|\underset{\mathcal{D}_{1}^{\prime}}{\operatorname{Pr}}[x]-\underset{\mathcal{D}_{2}^{\prime}}{\operatorname{Pr}}[x]\right|=\frac{1}{2} \sum_{x: E(x)}\left|\frac{p_{x}}{a}-\frac{q_{x}}{b}\right| \\
& \leq \frac{1}{2 b} \sum_{x: E(x)}\left(\left|p_{x}-q_{x}\right|+\left|p_{x}-\frac{b}{a} p_{x}\right|\right) \\
& \leq \frac{\kappa}{2 b}+\frac{1}{2}\left|1-\frac{a}{b}\right| \leq \frac{\kappa}{b} \leq \frac{\kappa}{a-\kappa} .
\end{aligned}
$$

By construction, every measurement outcome $|i\rangle \pm|j\rangle$ in the support of every $\mathcal{D}_{\left|\xi_{k}\right\rangle}^{\prime}$ corresponds to an edge $(i, j)$ that is $c$-unbalanced with respect to $\left|\varphi_{1}\right\rangle$. But this still leaves a key question unanswered: is $(i, j)$ reasonably unbalanced with respect to $\left|\xi_{k}\right\rangle$ itself? The following lemma will imply that it is, with high probability over $\mathcal{D}_{\left|\xi_{k}\right\rangle}^{\prime}$ : in particular that

$$
\operatorname{Pr}_{|i\rangle \pm|j\rangle \in \mathcal{D}_{\left|\xi_{k}\right\rangle}^{\prime}}\left[(i, j) \text { is } \frac{c}{4} \text {-unbalanced w.r.t. }\left|\xi_{k}\right\rangle\right] \geq 1-\frac{16 \sqrt{2 \delta}}{c(d-\sqrt{2 \delta})}
$$

for all $k \in\left[K^{\prime \prime}\right]$.

Lemma 3.15. Let $\mathcal{D}=\left(p_{x}, q_{x}\right)_{x \in[N]}$ and $\mathcal{D}^{\prime}=\left(p_{x}^{\prime}, q_{x}^{\prime}\right)_{x \in[N]}$ be any two probability distributions over the set $[N] \times\{0,1\}$. Suppose that $\left\|\mathcal{D}-\mathcal{D}^{\prime}\right\| \leq \mu$, and that $2 p_{x} q_{x} \geq c\left(p_{x}+q_{x}\right)^{2}$ for every $x \in[N]$. Let $\mathcal{S}$ be the set of all $x \in[N]$ such that $2 p_{x}^{\prime} q_{x}^{\prime} \geq c^{\prime}\left(p_{x}^{\prime}+q_{x}^{\prime}\right)^{2}$. Then

$$
\sum_{x \in \mathcal{S}}\left(p_{x}^{\prime}+q_{x}^{\prime}\right) \geq 1-\frac{8 \mu}{c-2 c^{\prime}}
$$

for all constants $c \in(0,1 / 2)$ and $c^{\prime} \in(0, c / 2)$.

Proof. Assume for simplicity that $p_{x}, q_{x}>0$ for all $x$ (it is not hard to remove this restriction). Let $\varepsilon_{x}=p_{x}^{\prime}-p_{x}$ and $\delta_{x}=q_{x}^{\prime}-q_{x}$. Then by assumption,

$$
\sum_{x=1}^{N}\left(\left|\varepsilon_{x}\right|+\left|\delta_{x}\right|\right) \leq 2 \mu
$$




\section{S. Aaronson, S. Beigi, A. Drucker, B. Fefferman, And P. Shor}

Let $\overline{\mathcal{S}}$ be the complement of $\mathcal{S}$. Consider an adversary with a "budget" of $2 \mu$, who is trying to perturb $\mathcal{D}$ so as to maximize $\sum_{x \in \overline{\mathcal{S}}}\left(p_{x}^{\prime}+q_{x}^{\prime}\right)$. Define the "price per pound" of $x$ to be

$$
\$_{x}:=\frac{\left|\varepsilon_{x}\right|+\left|\delta_{x}\right|}{p_{x}^{\prime}+q_{x}^{\prime}}
$$

Intuitively, $\$_{x}$ is the amount the adversary has to "spend" on perturbing $p_{x}$ and $q_{x}$, divided by the amount of probability mass that gets added to $\bar{S}$ as a result. We will show that $\$_{x} \geq\left(c-2 c^{\prime}\right) / 4$ for all $x \in \overline{\mathcal{S}}$. This will suffice to prove the lemma, since we then have

$$
\sum_{x \in \overline{\mathcal{S}}}\left(p_{x}^{\prime}+q_{x}^{\prime}\right)=\sum_{x \in \overline{\mathcal{S}}} \frac{\left|\varepsilon_{x}\right|+\left|\delta_{x}\right|}{\$_{x}} \leq \frac{8 \mu}{c-2 c^{\prime}} .
$$

We now lower-bound $\$_{x}$. If we simply divide through by $p_{x} q_{x}$, the condition $2 p_{x} q_{x} \geq c\left(p_{x}+q_{x}\right)^{2}$ is equivalent to $p_{x} / q_{x}+q_{x} / p_{x} \leq(2-2 c) / c$. Let $A=(2-2 c) / c$; then in particular, we have $p_{x} \leq A q_{x}$ and $q_{x} \leq A p_{x}$ for all $x$. On the other hand, to get $x \in \overline{\mathcal{S}}$ we need $p_{x}^{\prime} / q_{x}^{\prime}+q_{x}^{\prime} / p_{x}^{\prime}>\left(2-2 c^{\prime}\right) / c^{\prime}$, and hence either $p_{x}^{\prime} / q_{x}^{\prime}>B$ or $q_{x}^{\prime} / p_{x}^{\prime}>B$ where $B=\left(1-c^{\prime}\right) / c^{\prime}$.

Suppose $p_{x}^{\prime} / q_{x}^{\prime}>B$ without loss of generality. Then

$$
p_{x}+\varepsilon_{x}>B\left(q_{x}+\delta_{x}\right)>B\left(\frac{p_{x}}{A}+\delta_{x}\right),
$$

which rearranging means

$$
\varepsilon_{x}-B \delta_{x}>\left(\frac{B}{A}-1\right) p_{x}
$$

Likewise

$$
B\left(q_{x}+\delta_{x}\right)<p_{x}+\varepsilon_{x}<A q_{x}+\varepsilon_{x},
$$

which rearranging means

$$
\varepsilon_{x}-B \delta_{x}>(B-A) q_{x}>\left(\frac{B}{A}-1\right) q_{x}
$$

Combining,

$$
\varepsilon_{x}-B \delta_{x}>\left(\frac{B}{A}-1\right) \frac{p_{x}+q_{x}}{2}
$$

and hence

$$
\left|\varepsilon_{x}\right|+\left|\delta_{x}\right|>\frac{1}{B}\left(\varepsilon_{x}-B \delta_{x}\right)>\left(\frac{1}{A}-\frac{1}{B}\right) \frac{p_{x}+q_{x}}{2} .
$$

Therefore

$$
\begin{aligned}
\$_{x} & =\frac{\left|\varepsilon_{x}\right|+\left|\delta_{x}\right|}{p_{x}^{\prime}+q_{x}^{\prime}} \geq \frac{\left|\varepsilon_{x}\right|+\left|\delta_{x}\right|}{p_{x}+q_{x}+\left|\varepsilon_{x}\right|+\left|\delta_{x}\right|}>\frac{\frac{1}{2}(1 / A-1 / B)}{1+\frac{1}{2}(1 / A-1 / B)} \\
& =\frac{c /(2-2 c)-c^{\prime} /\left(1-c^{\prime}\right)}{2+c /(2-2 c)-c^{\prime} /\left(1-c^{\prime}\right)}=\frac{c-2 c^{\prime}+c c^{\prime}}{4-3 c-6 c^{\prime}+5 c c^{\prime}} \geq \frac{c-2 c^{\prime}}{4}
\end{aligned}
$$

as claimed. 
We now need one last conditioning step: discard all states $\left|\xi_{k}\right\rangle$ for which the measurement outcome is not $c / 4$-unbalanced with respect to $\left|\xi_{k}\right\rangle$. By Lemma 3.15, with overwhelming probability this still leaves us with $K^{\prime \prime \prime} \approx K^{\prime \prime}$ states (for suitable choices of $c, d$, and $\delta$ ). Call those states $\left|\varsigma_{1}\right\rangle, \ldots,\left|\varsigma_{K^{\prime \prime \prime}}\right\rangle$.

Given any state $|\varphi\rangle$, let $\mathcal{D}_{|\varphi\rangle}^{\prime \prime}$ be the probability distribution over $|i\rangle \pm|j\rangle \in \widetilde{\mathcal{M}}$ obtained by starting from $\mathcal{D}_{|\varphi\rangle}^{\prime}$, and then conditioning on the edge $(i, j)$ being $c / 4$-unbalanced with respect to $|\varphi\rangle$. Then

$$
\begin{aligned}
\left\|\mathcal{D}_{\left|\zeta_{k}\right\rangle}^{\prime \prime}-\mathcal{D}_{\left|\varphi_{1}\right\rangle}^{\prime}\right\| & \leq\left\|\mathcal{D}_{\left|\zeta_{k}\right\rangle}^{\prime \prime}-\mathcal{D}_{\left|\zeta_{k}\right\rangle}^{\prime}\right\|+\left\|\mathcal{D}_{\left|\zeta_{k}\right\rangle}^{\prime}-\mathcal{D}_{\left|\varphi_{1}\right\rangle}^{\prime}\right\| \\
& \leq \frac{16 \sqrt{2 \delta}}{c(d-\sqrt{2 \delta})}+\frac{\sqrt{2 \delta}}{d-\sqrt{2 \delta}} \\
& \leq \frac{17 \sqrt{2 \delta}}{c(d-\sqrt{2 \delta})}
\end{aligned}
$$

for all $k \in\left[K^{\prime \prime \prime}\right]$. So by the triangle inequality,

$$
\left\|\mathcal{D}_{\left|\varsigma_{k}\right\rangle}^{\prime \prime}-\mathcal{D}_{\left|\varsigma_{\ell}\right\rangle}^{\prime \prime}\right\| \leq \frac{34 \sqrt{2 \delta}}{c(d-\sqrt{2 \delta})}
$$

for all $k, \ell \in\left[K^{\prime \prime \prime}\right]$.

So to sum up: we have $K^{\prime \prime \prime}=\Theta(\sqrt{N})$ samples from $\widetilde{\mathcal{M}}$, drawn independently from probability distributions $\mathcal{D}_{\left|\varsigma_{1}\right\rangle}^{\prime \prime}, \ldots, \mathcal{D}_{\left|\zeta_{K^{\prime \prime \prime}}\right\rangle}^{\prime \prime}$ respectively. The distributions $\mathcal{D}_{\left|\varsigma_{k}\right\rangle}^{\prime \prime}$ have bounded variation distance from one another. We also know, because the $\mathcal{D}_{\left|\zeta_{k}\right\rangle}^{\prime \prime}$ 's only involve $c / 4$-unbalanced edges $(i, j)$, that if Arthur finds a collision among the $K^{\prime \prime \prime}$ samples (i. e., two samples of the form $|i\rangle \pm|j\rangle$ for some $(i, j)$ ), then that collision will also be a disagreement with constant probability. Thus, the one remaining task is to show that Arthur finds a collision with constant probability.

Showing this amounts to generalizing the Birthday Paradox still further, to the case where the birthday distributions are not only nonuniform but can also differ from each other by small amounts. In particular we want the following:

Theorem 3.16. Let $X_{1}, \ldots, X_{K}$ be independent random variables over $[N]$, and let $\mathcal{D}_{i}$ be the distribution over $X_{i}$. Suppose $K \geq 32 \sqrt{N}$ and $\left\|\mathcal{D}_{i}-\mathcal{D}_{j}\right\| \leq 1 / 10$ for all $i, j$. Then

$$
\operatorname{Pr}\left[\exists i, j: X_{i}=X_{j}\right] \geq \frac{1}{2}
$$

In Section 3.6, we present a proof of Theorem 3.16 based on the second moment method. (Indeed, our proof works even if the $X_{i}$ 's are only 4-wise independent.)

By Theorem 3.16, Arthur will find a collision among the $K^{\prime \prime \prime}=\Theta(\sqrt{N})$ remaining samples with constant probability. Then by the definition of the $\mathcal{D}_{\left|\varsigma_{k}\right\rangle}^{\prime \prime}$ 's, this collision will be a disagreement with constant probability, thereby causing Arthur to reject.

So in summary, we get a protocol with perfect completeness, constant soundness, and $\widetilde{O}(\sqrt{m})$ unentangled witnesses with $O(\log m)$ qubits each. 


\section{S. Aaronson, S. Beigi, A. Drucker, B. FefFerman, And P. Shor}

As a final remark, we can reduce the soundness error to be negligibly small (in particular, $2^{-\operatorname{polylog} m}$ ). To do so, we simply multiply the number of Merlins by a further polylog $m$ factor, and repeat the whole protocol polylog $m$ times.

\subsection{The generalized birthday paradox}

The purpose of this section is to prove the Birthday Paradox, even in the very general situation where

(1) the distributions over birthdays need not be uniform, and

(2) the distributions need not be the same for every person, but only $\varepsilon$-close in variation distance, and

(3) the distributions need not be independent, but only 4-wise independent.

First we need two lemmas.

Lemma 3.17. Let $\mathcal{D}_{1}, \mathcal{D}_{2}$ be probability distributions over $[n]$ such that $\left\|\mathcal{D}_{1}-\mathcal{D}_{2}\right\| \leq \varepsilon$. Then

$$
\underset{x \in \mathcal{D}_{1}, y \in \mathcal{D}_{2}}{\operatorname{Pr}}[x=y] \geq(1-\varepsilon)^{2} / n .
$$

Proof. Let $p_{x}=\operatorname{Pr}_{\mathcal{D}_{1}}[x]$ and let $q_{x}=\operatorname{Pr}_{\mathcal{D}_{2}}[x]$. Then

$$
\operatorname{Pr}_{x \in \mathcal{D}_{1}, y \in \mathcal{D}_{2}}[x=y]=\sum_{x \in[n]} p_{x} q_{x} \geq \sum_{x \in[n]} \min \left(p_{x}, q_{x}\right)^{2} \geq \frac{1}{n}\left(\sum_{x \in[n]} \min \left(p_{x}, q_{x}\right)\right)^{2}=\frac{1}{n}(1-\varepsilon)^{2}
$$

where the second inequality follows from Cauchy-Schwarz.

Lemma 3.18. Let $p_{1}, \ldots, p_{K}$ be nonnegative reals, and let $r=\sum_{i<j<k} p_{i} p_{j} p_{k}$ and $s=\sum_{i<j} p_{i} p_{j}$. Then $r^{2} \leq 2 s^{3}$.

Proof. Let $\mathcal{S}$ be the set of 6-tuples $(i, j, k, \ell, m, n)$ such that $i<j, k<\ell$, and $m<n$, and let $\mathcal{R}$ be the set of 6-tuples such that $i<j<k$ and $\ell<m<n$. Then

$$
s^{3}=\sum_{\mathcal{S}} p_{i} p_{j} p_{k} p_{\ell} p_{m} p_{n}
$$

while

$$
r^{2}=\sum_{\mathcal{R}} p_{i} p_{j} p_{k} p_{\ell} p_{m} p_{n}
$$

Now define a mapping from $\mathcal{R}$ to $\mathcal{S}$, by simply swapping $k$ and $\ell$ if $k>\ell$, or swapping $\ell$ and $m$ if $k=\ell$. It is easily checked that this mapping is two-to-one. Hence $r^{2} \leq 2 s^{3}$ as claimed.

We now prove Theorem 3.16, which we restate for convenience.

Theorem. Let $X_{1}, \ldots, X_{K}$ be 4-wise independent random variables over $[n]$, and let $\mathcal{D}_{i}$ be the marginal distribution over $X_{i}$. Suppose $K \geq 32 \sqrt{n}$ and $\left\|\mathcal{D}_{i}-\mathcal{D}_{j}\right\| \leq 1 / 10$ for all $i, j$. Then

$$
\operatorname{Pr}\left[\exists i, j: X_{i}=X_{j}\right] \geq \frac{1}{2}
$$


Proof. Let $Y_{i j}$ be 1 if $X_{i}=X_{j}$ and 0 otherwise, and let $Y:=\sum_{i<j} Y_{i j}$. By Lemma 3.17, we have

$$
\mathrm{E}[Y] \geq\left(\begin{array}{l}
K \\
2
\end{array}\right) \frac{(1-1 / 10)^{2}}{n} \geq 900 .
$$

The remainder of the proof will involve upper-bounding the second moment $\mathrm{E}\left[Y^{2}\right]$. Let us write

$$
\mathrm{E}\left[Y^{2}\right]=\sum_{i<j, k<\ell} \mathrm{E}\left[Y_{i j} Y_{k \ell}\right]=\tau_{2}+\tau_{3}+\tau_{4}
$$

where $\tau_{N}$ contains the terms in which $N$ distinct indices appear among $\{i, j, k, l\}$. It is easy to see that

$$
\tau_{2}=\sum_{i<j} \mathrm{E}\left[Y_{i j}\right]=\mathrm{E}[Y]
$$

and (by 4-wise independence) that

$$
\tau_{4}=\sum_{\substack{i<j, k<l \\ \text { all distinct }}} \mathrm{E}\left[Y_{i j}\right] \mathrm{E}\left[Y_{k \ell}\right] \leq \mathrm{E}[Y]^{2} .
$$

So the nontrivial part is to upper-bound $\tau_{3}$. Let $p_{i, x}:=\operatorname{Pr}\left[X_{i}=x\right]$. Also, let

$$
\begin{aligned}
r_{x} & :=\sum_{i<j<k} p_{i, x} p_{j, x} p_{k, x}, \\
s_{x} & :=\sum_{i<j} p_{i, x} p_{j, x}
\end{aligned}
$$

and notice that $\sum_{x \in[n]} s_{x}=\mathrm{E}[Y]$. Then

$$
\begin{aligned}
\tau_{3} & =6 \sum_{i<j<k} \sum_{x \in[n]} p_{i, x} p_{j, x} p_{k, x} \\
& =6 \sum_{x \in[n]} r_{x} \\
& \leq 6 \sum_{x \in[n]} \sqrt{2 s_{x}^{3}} \\
& \leq 6 \sqrt{2} \sum_{x \in[n]}\left(\frac{1}{40} s_{x}^{2}+12 s_{x}\right) \\
& \leq 6 \sqrt{2}\left(\frac{1}{40}\left(\sum_{x \in[n]} s_{x}\right)^{2}+12 \sum_{x \in[n]} s_{x}\right) \\
& =6 \sqrt{2}\left(\frac{\mathrm{E}[Y]^{2}}{40}+12 \mathrm{E}[Y]\right) .
\end{aligned}
$$


Here the third line follows from Lemma 3.18, and the fourth line follows from the basic calculus fact that $s^{3 / 2} \leq \frac{1}{40} s^{2}+12 s$ for all nonnegative $s$. Hence

$$
\begin{aligned}
\operatorname{Pr}[Y=0] & \leq \operatorname{Pr}[|Y-\mathrm{E}[Y]| \geq \mathrm{E}[Y]]=\operatorname{Pr}\left[(Y-\mathrm{E}[Y])^{2} \geq \mathrm{E}[Y]^{2}\right] \leq \frac{\operatorname{Var}[Y]}{\mathrm{E}[Y]^{2}} \\
& =\frac{\mathrm{E}\left[Y^{2}\right]-\mathrm{E}[Y]^{2}}{\mathrm{E}[Y]^{2}}=\frac{\tau_{2}+\tau_{3}+\tau_{4}-\mathrm{E}[Y]^{2}}{\mathrm{E}[Y]^{2}} \\
& \leq \frac{\mathrm{E}[Y]+6 \sqrt{2}\left(\mathrm{E}[Y]^{2} / 40+12 \mathrm{E}[Y]\right)+\mathrm{E}[Y]^{2}-\mathrm{E}[Y]^{2}}{\mathrm{E}[Y]^{2}} \\
& =\frac{1+72 \sqrt{2}}{\mathrm{E}[Y]}+\frac{6 \sqrt{2}}{40} \leq \frac{1}{2}
\end{aligned}
$$

Finally,

$$
\operatorname{Pr}\left[\exists i, j: X_{i}=X_{j}\right]=1-\operatorname{Pr}[Y=0] \geq \frac{1}{2}
$$

and we are done.

\subsection{General observations}

We conclude this subsection by making three general observations about Theorem 1.1.

First, we strongly believe that our protocol can be improved to one involving two provers, one of whom sends $O(\log m)$ qubits and the other of whom sends $O(\sqrt{m}$ polylog $m)$ qubits. Specifically, if all but one of the witnesses in our current protocol are entangled with one another, in a way that breaks the protocol's soundness, we believe Arthur should be able to use the remaining witness to detect this. This is a problem we leave to future work.

Second, our protocol made essential use of the PCP Theorem, in the strong version proved by Dinur [11]. One might wonder whether Theorem 1.1 could also be proved in a "black-box" fashion, without exploiting anything about the structure of 3SAT. The following simple result shows that the answer is no-and that indeed, in the black-box setting, there is essentially no savings at all over the classical witness size.

Theorem 3.19. Let $f:\{0,1\}^{n} \rightarrow\{0,1\}$ be a black-box function. Then any $\mathrm{QMA}^{f}(k)$ protocol to convince Arthur that there exists an $x$ such that $f(x)=1$, with soundness gap $\Omega(1 / \operatorname{poly}(n))$, must involve $n-O(\log n)$ qubits sent by the Merlins.

Proof Sketch. Assume without loss of generality that either $f$ is identically zero, or else there exists a unique "marked item" $x^{*}$ such that $f\left(x^{*}\right)=1$. Suppose it were possible to convince Arthur that $x^{*}$ exists by giving him unentangled witnesses $\left|\varphi_{1}\right\rangle, \ldots,\left|\varphi_{K}\right\rangle$ with $Q$ qubits in total. Then given these witnesses, Arthur's verification algorithm must query $f\left(x^{*}\right)$ at some time step with non-negligible probability $\beta=$ $\Omega(1 / \operatorname{poly}(n))$. For otherwise, by the hybrid argument of Bennett, Bernstein, Brassard, and Vazirani [4], Arthur's verification algorithm would not have $\Omega(1 /$ poly $(n))$ soundness (i. e., Arthur would fail to detect a change in $f\left(x^{*}\right)$ from 1 to 0 ). But this means that Arthur's algorithm can be modified to one that 
uses no witnesses, and that finds $x^{*}$ with probability at least $2^{-Q} \beta / T$. For Arthur can simply replace $\left|\varphi_{1}\right\rangle, \ldots,\left|\varphi_{K}\right\rangle$ by the $Q$-qubit maximally mixed state, then measure at a random time step to find which $x$ is being queried. On the other hand, we know from the result of Bennett et al. [4] mentioned previously that if $x^{*}$ is uniformly random, then after $T$ queries, Arthur can have found $x^{*}$ with probability at most $4 T^{2} / 2^{n}$. Solving $2^{-Q} \beta / T \leq 4 T^{2} / 2^{n}$ for $Q$, we find that

$$
Q \geq \log \frac{\beta 2^{n}}{4 T^{3}} \geq n-O(\log n) .
$$

Third, notice that our protocol does not let Arthur find a satisfying assignment for $\varphi$; it only convinces him that such an assignment exists. If there were a way to modify our protocol to let Arthur recover an assignment, this would have a spectacular consequence for quantum algorithms. Namely, by running Arthur's verification procedure with the $\widetilde{O}(\sqrt{m})$-qubit maximally mixed state in place of the witnesses, we could find a satisfying assignment for $\varphi$ with probability $2^{-\widetilde{O}(\sqrt{m})}$, with no help from any Merlins. But this would yield a $2^{\widetilde{O}(\sqrt{m})}$-time quantum algorithm for $3 \mathrm{SAT}$-and in particular, a $2^{\widetilde{O}(\sqrt{n})}$-time algorithm in the "critical regime" $m=O(n)$ !

\section{Weak additivity implies amplification}

In this section we show how to amplify any $\mathrm{QMA}(k)$ protocol to exponentially small error, and to simulate $k$ provers with two, assuming a weak version of the Additivity Conjecture.

\subsection{Entanglement of formation}

The analysis of our amplification protocol will involve showing that Arthur cannot create "too much" entanglement during his verification procedure. To make this precise, we need some way to measure the entanglement of mixed states. Fortunately, this is one of the most studied topics in all of quantum information theory. One particular entanglement measure- the entanglement of formation $E_{F}$ defined by Bennett et al. [6]—will be particularly useful for us.

To define $E_{F}$ we first need some other concepts. Given a mixed state $\sigma$, the von Neumann entropy of $\sigma$ is $S(\sigma):=H\left(\left\{\lambda_{i}\right\}\right)$, where $H\left(\left\{p_{i}\right\}\right)=-\sum_{i} p_{i} \log _{2} p_{i}$ is the usual entropy function and $\left\{\lambda_{i}\right\}$ are the eigenvalues of $\sigma$. Given a bipartite pure state $\left|\psi^{A B}\right\rangle$, let $\sigma^{A}$ and $\sigma^{B}$ be the reduced states of the $A$ and $B$ registers respectively. Then it is not hard to show that $S\left(\sigma^{A}\right)=S\left(\sigma^{B}\right)$. We call this quantity the entanglement entropy of $\left|\psi^{A B}\right\rangle$, or $E\left(\left|\psi^{A B}\right\rangle\right)$. We can then define $E_{F}\left(\rho^{A B}\right)$ as a weighted average of entanglement entropy, minimized over all purifications of $\rho^{A B}$ :

Definition 4.1. Given a bipartite state $\rho^{A B}$, the entanglement of formation $E_{F}\left(\rho^{A B}\right)$ is the minimum of $\sum_{i} p_{i} E\left(\left|\psi_{i}\right\rangle\right)$ over all decompositions $\rho^{A B}=\sum_{i} p_{i}\left|\psi_{i}\right\rangle\left\langle\psi_{i}\right|$.

Intuitively, $E_{F}$ measures the minimum number of entangled pairs $\frac{1}{\sqrt{2}}(|00\rangle+|11\rangle)$ that are needed to prepare $\rho^{A B}$. 


\section{S. Aaronson, S. Beigi, A. Drucker, B. Fefferman, And P. Shor}

Almost by definition, $E_{F}$ satisfies convexity: for all $\rho^{A B}$ and $\sigma^{A B}$,

$$
E_{F}\left(p \rho^{A B}+(1-p) \sigma^{A B}\right) \leq p E_{F}\left(\rho^{A B}\right)+(1-p) E_{F}\left(\sigma^{A B}\right) .
$$

It is also easy to see that $E_{F}\left(\rho^{A B}\right)=0$ if and only if $\rho^{A B}$ is separable. In this paper, we will need two further properties of $E_{F}$. The first property is what we called "faithfulness" in Section 1.4.

Lemma 4.2. Suppose $E_{F}\left(\rho^{A B}\right) \leq \varepsilon$. Then there exists a separable state that is $\sqrt{2 \varepsilon}$-close to $\rho^{A B}$ in trace distance.

Proof. Let $S(\rho \| \sigma)$ be the quantum relative entropy between mixed states $\rho$ and $\sigma$ (see Nielsen and Chuang [23] for a definition). Then Vedral and Plenio [28] showed that

$$
E_{F}\left(\rho^{A B}\right) \geq \min S\left(\rho^{A B} \| \sigma^{A B}\right),
$$

where the minimum is taken over all separable states $\sigma^{A B}$. Also, it is known (see Klauck et al. [16] and Ohya and Petz [24]) that

$$
S\left(\rho^{A B} \| \sigma^{A B}\right) \geq \frac{1}{2}\left\|\rho^{A B}-\sigma^{A B}\right\|_{\text {tr }}^{2} .
$$

Putting these results together, if $E_{F}\left(\rho^{A B}\right) \leq \varepsilon$ then there exists a separable state $\sigma^{A B}$ such that

$$
S\left(\rho^{A B} \| \sigma^{A B}\right) \leq \varepsilon
$$

and hence $\left\|\rho^{A B}-\sigma^{A B}\right\|_{\text {tr }} \leq \sqrt{2 \varepsilon}$.

The second property is that, if we start from a separable state, we cannot increase the value of $E_{F}$ much by measuring few qubits and then conditioning on the outcome.

Lemma 4.3. Let $\rho^{A B}$ be a separable state, and suppose $\sigma^{A B}$ is obtained from $\rho^{A B}$ by applying an arbitrary entangled measurement on at most $n$ qubits from each register, and then possibly conditioning on the outcome. Then $E_{F}\left(\sigma^{A B}\right) \leq E_{F}\left(\rho^{A B}\right)+2 n$.

Proof. By convexity, we can assume without loss of generality that $\rho^{A B}$ is a pure state, $\left|\psi_{A}\right\rangle \otimes\left|\psi_{B}\right\rangle$. So we can write $\sigma^{A}$ as

$$
\frac{\Phi\left(\left|\psi_{A}\right\rangle\left\langle\psi_{A}\right|\right)}{\left\|\Phi\left(\left|\psi_{A}\right\rangle\left\langle\psi_{A}\right|\right)\right\|},
$$

where $\Phi$ is some non-trace-increasing operator acting on at most $n$ qubits. Or in the operator-sum representation,

$$
\sigma^{A}=\frac{\sum_{i=1}^{M} E_{i}\left|\psi_{A}\right\rangle\left\langle\psi_{A}\right| E_{i}^{\dagger}}{\operatorname{Tr} \sum_{i=1}^{M} E_{i}\left|\psi_{A}\right\rangle\left\langle\psi_{A}\right| E_{i}^{\dagger}}
$$

where $\sum_{i=1}^{2^{2 n}} E_{i}^{\dagger} E_{i} \preceq I$ and $M \leq 2^{2 n}$. We then have

$$
\begin{aligned}
E_{F}\left(\sigma^{A B}\right) & \leq S\left(\sigma^{A}\right) \\
& =S\left(\frac{\sum_{i=1}^{M} E_{i}\left|\psi_{A}\right\rangle\left\langle\psi_{A}\right| E_{i}^{\dagger}}{\operatorname{Tr} \sum_{i=1}^{M} E_{i}\left|\psi_{A}\right\rangle\left\langle\psi_{A}\right| E_{i}^{\dagger}}\right) \\
& \leq \log _{2} M \leq 2 n
\end{aligned}
$$

where the first line follows from the concavity of the von Neumann entropy. 
Given an entanglement measure $E$, we call $E$ superadditive if for any state $\rho^{A A^{\prime}, B B^{\prime}}$ on four registers,

$$
E\left(\rho^{A A^{\prime}, B B^{\prime}}\right) \geq E\left(\rho^{A B}\right)+E\left(\rho^{A^{\prime} B^{\prime}}\right) .
$$

As mentioned earlier, the analysis of our QMA $(k)$ amplification protocol originally relied on the conjecture that $E_{F}$ is superadditive. But in a spectacular recent development, Hastings [13] has shown that this conjecture is false. (More precisely, Hastings shows a failure of additivity for the minimum output entropy of a quantum channel. By a result of Shor [27], this is equivalent to the superadditivity of entanglement of formation.)

However, the violation of additivity found by Hastings is extremely small, and is perfectly consistent with additivity being true in a weaker or asymptotic sense. To make this precise, we now state a version of the Additivity Conjecture that suffices for our purposes. Call an entanglement measure $E$ weakly superadditive if it satisfies the relation

$$
E\left(\rho^{A_{1} A_{2} \cdots A_{k}, B_{1} B_{2} \cdots B_{k}}\right) \geq \frac{c}{k} \sum_{i, j=1}^{k} E\left(\rho^{A_{i} B_{j}}\right),
$$

for some constant $c$ independent of $k$. Weak superadditivity is, in particular, implied by the following inequality:

$$
E\left(\rho^{A A^{\prime}, B B^{\prime}}\right) \geq \frac{1}{2}\left[E\left(\rho^{A B}\right)+E\left(\rho^{A B^{\prime}}\right)+E\left(\rho^{A^{\prime} B}\right)+E\left(\rho^{A^{\prime} B^{\prime}}\right)\right]
$$

which in turn is implied by ordinary superadditivity. Then we conjecture the following:

Conjecture 4.4 (Weak Additivity Conjecture). $E_{F}$ is weakly superadditive.

As a side note, $E_{F}$ badly violates the so-called monogamy inequality ${ }^{11}$

$$
E\left(\rho^{A, B B^{\prime}}\right) \geq E\left(\rho^{A B}\right)+E\left(\rho^{A B^{\prime}}\right) .
$$

As an example, consider again the maximally antisymmetric state

$$
|\psi\rangle=\frac{1}{\sqrt{N !}} \sum_{\sigma \in S_{N}}(-1)^{\operatorname{sgn}(\sigma)}|\sigma(1)\rangle \cdots|\sigma(N)\rangle .
$$

The entanglement of formation between the first register of $|\psi\rangle$ and the remaining $N-1$ registers is at $\operatorname{most} \log N$. Yet the entanglement of formation between the first register and any one other register can be shown to be $\Omega(1)$. Note that, had $E_{F}$ satisfied the monogamy inequality, we would have been able to use it to show $\mathrm{QMA}(2)=\mathrm{QMA}$, using essentially the same argument as in footnote 3 .

A different entanglement measure - the squashed entanglement $E_{s q}$ of Christandl and Winter [10]is known to satisfy both superadditivity and the stronger monogamy inequality. The trouble with $E_{s q}$ is that it badly violates the analogue of Lemma 4.2: there exist $N \times N$-dimensional bipartite states $\rho^{A B}$ Such

\footnotetext{
${ }^{11}$ Note that the monogamy inequality implies superadditivity, via

$$
E\left(\rho^{A A^{\prime}, B B^{\prime}}\right) \geq E\left(\rho^{A A^{\prime}, B}\right)+E\left(\rho^{A A^{\prime}, B^{\prime}}\right) \geq E\left(\rho^{A B}\right)+E\left(\rho^{A^{\prime} B}\right)+E\left(\rho^{A B^{\prime}}\right)+E\left(\rho^{A^{\prime} B^{\prime}}\right) \geq E\left(\rho^{A B}\right)+E\left(\rho^{A^{\prime} B^{\prime}}\right) .
$$
}




\section{S. Aaronson, S. Beigi, A. Drucker, B. Fefferman, And P. Shor}

that $E_{s q}\left(\rho^{A B}\right)=O\left(\frac{\log N}{N}\right)$, yet $\rho^{A B}$ has trace distance $\Omega(1)$ to any separable state. The example that shows this is once again the maximally antisymmetric state, which seems like the "universal counterexample" of entanglement theory! This is why we cannot use squashed entanglement in this paper, and must instead use entanglement of formation.

\subsection{The two-prover case}

We now show that the Weak Additivity Conjecture implies the QMA(2) Amplification Conjecture.

Theorem 4.5. Assume the Weak Additivity Conjecture. Then $\mathrm{QMA}(2, a, b)=\mathrm{QMA}\left(2,2^{-p(n)}, 1-2^{-p(n)}\right)$ for all $b-a=\Omega(1 / \operatorname{poly}(n))$ and all polynomials $p$.

Proof. Let $L$ be a language in $\operatorname{QMA}(2, a, b)$; then we need to show $L \in \mathrm{QMA}\left(2,2^{-p(n)}, 1-2^{-p(n)}\right)$. Let $Q$ be Arthur's verification algorithm in the original QMA $(2, a, b)$ protocol, and let the original Merlins' messages have $r(n)$ qubits each for some polynomial $r$. Also, let $T(n)$ be a number of repetitions of $Q$ that suffices to amplify it to error probability $2^{-p(n)}$, assuming no entanglement among $\operatorname{Merlin}_{A}$ 's or $\operatorname{Merlin}_{B}$ 's registers. By a standard Chernoff bound, we can take $T(n):=C \cdot p(n) /(b-a)^{2}$ for some constant $C$.

Our amplified protocol is the following.

(1) Arthur asks Merlin ${ }_{A}$ and $\operatorname{Merlin}_{B}$ to supply $q(n)$ copies each of their respective witnesses, where $q(n):=C^{\prime} \cdot T(n) r(n) /(b-a)^{2}$ for some constant $C^{\prime}$. Denote by $\rho^{A_{1} A_{2} \cdots A_{q(n)}}$ and $\rho^{B_{1} B_{2} \cdots B_{q(n)}}$ the states on $q(n) r(n)$ qubits that Arthur actually receives.

(2) For all $t:=1$ to $T(n)$, Arthur chooses registers $A_{j}$ and $B_{k}$ uniformly and independently from among those not already chosen, and runs $Q$ on the state $\rho^{A_{j} B_{k}}$.

(3) Arthur accepts if at least $\frac{a+b}{2} T(n)$ of the $T(n)$ invocations of $Q$ accepted, and rejects otherwise.

We need to show two things about this protocol, completeness and soundness.

Completeness: If the Merlins are honest, they can simply send $\left|\psi_{A}\right\rangle^{\otimes q(n)}$ and $\left|\psi_{B}\right\rangle^{\otimes q(n)}$ respectively, where $\left|\psi_{A}\right\rangle \otimes\left|\psi_{B}\right\rangle$ is a witness that $Q$ accepts with probability at least $b$. Then by assumption, Arthur will accept with probability at least $1-2^{-p(n)}$.

Soundness: As usual, this is the interesting part. Our central claim is the following: At every one of the $T(n)$ iterations, Arthur can be considered to be running $Q$ on a bipartite state $\rho^{A B}$ that is $\varepsilon$-close to a separable state, where $\varepsilon=O(\sqrt{T(n) r(n) / q(n)})$.

Let us first see why soundness follows from the above claim. Suppose $x \notin L$. Then $Q$ accepts every separable state with probability at most $a$. By Proposition 2.6, then, $Q$ also accepts every state that is $\varepsilon$-close to separable with probability at most $a+\varepsilon$. But

$$
\varepsilon=O\left(\sqrt{\frac{T(n) r(n)}{q(n)}}\right) \leq \frac{b-a}{4},
$$


provided we chose a sufficiently large constant $C^{\prime}$ when defining $q(n)$. So every invocation of $Q$ accepts with probability at most $a+(b-a) / 4$. Therefore, provided we choose a sufficiently large constant $C$ when defining $T(n)$, Arthur will accept with probability at most $2^{-p(n)}$ by a Chernoff bound.

We now prove the claim. By Lemma 4.3, the entanglement of formation between Merlin ${ }_{A}$ 's registers and $\operatorname{Merlin}_{B}$ 's registers can be at most $2 r(n)$ after the first iteration, at most $4 r(n)$ after the second iteration, and so on. Hence

$$
E_{F}\left(\rho^{A_{1} A_{2} \cdots A_{q(n)}, B_{1} B_{2} \cdots B_{q(n)}}\right) \leq 2 T(n) r(n)
$$

throughout the protocol. Also, let $S_{A}$ and $S_{B}$ be the sets of $A$-registers and $B$-registers respectively that Arthur has not yet chosen. Then $\left|S_{A}\right|=\left|S_{B}\right|=q(n)-T(n)$. Assuming the Weak Additivity Conjecture, we therefore have

$$
\begin{aligned}
\sum_{A_{j} \in S_{A}, B_{k} \in S_{B}} E_{F}\left(\rho^{A_{j} B_{k}}\right) & =O\left((q(n)-T(n)) E_{F}\left(\rho^{\left.A_{1} A_{2} \cdots A_{q(n)}, B_{1} B_{2} \cdots B_{q(n)}\right)}\right)\right) \\
& =O(T(n) r(n)(q(n)-T(n))) .
\end{aligned}
$$

So if we define

$$
\sigma:=\frac{1}{\left|S_{A}\right|\left|S_{B}\right|} \sum_{A_{j} \in S_{A}, B_{k} \in S_{B}} \rho^{A_{j} B_{k}}
$$

then the convexity of $E_{F}$ implies that

$$
E_{F}(\sigma) \leq \frac{1}{\left|S_{A}\right|\left|S_{B}\right|} \sum_{A_{j} \in S_{A}, B_{k} \in S_{B}} E_{F}\left(\rho^{A_{j} B_{k}}\right)=O\left(\frac{T(n) r(n)}{q(n)-T(n)}\right)=O\left(\frac{T(n) r(n)}{q(n)}\right),
$$

using the fact that $T(n) \leq q(n) / 2$. By Lemma 4.2, this means that $\sigma$ is $O(\sqrt{T(n) r(n) / q(n)})$-close to a separable state, as claimed.

\subsection{The $k$-prover case}

In this section we show unconditionally that any $\mathrm{QMA}(k)$ protocol with constant soundness can be simulated by a $\mathrm{QMA}(2)$ protocol with soundness $\Omega(1 / k)$. Combined with Theorem 4.5 , this will imply that assuming the Weak Additivity Conjecture, for all $k \geq 2$ we have

$$
\mathrm{QMA}(k, 1 / 3,2 / 3) \subseteq \mathrm{QMA}(2, a, b) \subseteq \mathrm{QMA}(2,1 / 3,2 / 3)
$$

for some $a, b$ with $b-a=\Omega(1 / k)$, and hence QMA $(k)=\mathrm{QMA}(2)$. Note that Kobayashi et al. (personal communication) have independently shown that amplification of QMA (2) protocols implies $\mathrm{QMA}(k)=$ QMA(2) for all $k \geq 2$. (Their earlier result only showed that amplification of QMA $(k)$ protocols implies $\mathrm{QMA}(k)=\mathrm{QMA}(2)$ for all $k \geq 2$, which was not strong enough for our purposes.)

Theorem 4.6. $\mathrm{QMA}(k, a, b) \subseteq \mathrm{QMA}\left(2,1-\frac{(b-a)^{2}}{8 k}, 1-2^{-n}\right)$. 


\section{S. Aaronson, S. Beigi, A. Drucker, B. Fefferman, And P. Shor}

Proof. We will show that for all $k$ and all $\delta=\Omega(1 / \operatorname{poly}(n))$,

$$
\mathrm{QMA}\left(k, 1-\delta, 1-2^{-n}\right) \subseteq \mathrm{QMA}\left(2,1-\frac{\delta^{2}}{8 k}, 1-2^{-n}\right)
$$

This will suffice to prove the theorem, since Lemma 2.5 implies that for all $k$ and all $a, b$, we have $\mathrm{QMA}(k, a, b) \subseteq \mathrm{QMA}\left(k, 1-(b-a), 1-2^{-n}\right)$.

Our protocol is as follows. Merlin ${ }_{A}$ and $\operatorname{Merlin}_{B}$ send $k$-partite states $\rho^{A_{1} A_{2} \cdots A_{k}}$ and $\rho^{B_{1} B_{2} \cdots B_{k}}$ respectively. Given these states, Arthur performs one of the following two tests, each with probability $1 / 2$ :

(1) Choose $i \in[k]$ uniformly at random, perform a swap test between $\rho^{A_{i}}$ and $\rho^{B_{i}}$, and accept if and only if the swap test accepts.

(2) Simulate the $\mathrm{QMA}\left(k, 1-\delta, 1-2^{-n}\right)$ protocol, using $\rho^{A_{1} A_{2} \cdots A_{k}}$ in place of the $k$ witness registers.

We first show completeness of the above protocol. If the Merlins are honest, they can both simply send $k$ unentangled accepting witnesses for the $\mathrm{QMA}(k)$ protocol being simulated. In that case step (1) accepts with probability 1 , while step (2) accepts with probability at least $1-2^{-n}$.

We now show soundness. Suppose any set of unentangled witnesses causes the QMA $(k)$ protocol to reject with probability at least $\delta$. Then we need to show that any pair of witnesses $\rho^{A_{1} A_{2} \cdots A_{k}}$ and $\rho^{B_{1} B_{2} \cdots B_{k}}$ causes the QMA $(2)$ protocol to reject with probability at least $\delta^{2} /(8 k)$. We consider two cases.

First suppose $\rho^{A_{1} A_{2} \cdots A_{k}}$ is $\varepsilon$-close in trace distance to some separable pure state $|\Psi\rangle$. Then by Proposition 2.6, step (2) rejects with probability at least $\delta-\varepsilon$.

Next suppose $\rho^{A_{1} A_{2} \cdots A_{k}}$ is $\varepsilon$-far in trace distance from any separable pure state. Then by Proposition 2.9, we have $\left\langle\Psi\left|\rho^{A_{1} A_{2} \cdots A_{k}}\right| \Psi\right\rangle<1-\varepsilon^{2}$ for all separable pure states $|\Psi\rangle$. So taking the contrapositive of Proposition 2.8, for all pure states $\left|\psi_{1}\right\rangle, \ldots,\left|\psi_{k}\right\rangle$ we have

$$
\sum_{i=1}^{k}\left(1-\left\langle\psi_{i}\left|\rho^{A_{i}}\right| \psi_{i}\right\rangle\right)>\varepsilon^{2}
$$

Hence step (1) rejects with probability greater than $\varepsilon^{2} /(2 k)$ by Proposition 2.10 . Setting $\varepsilon=3 \delta / 4$, we thus find that the protocol rejects with probability at least

$$
\frac{1}{2} \max \left\{\frac{\delta}{4}, \frac{(3 \delta / 4)^{2}}{2 k}\right\} \geq \frac{\delta^{2}}{8 k} .
$$

Combining Theorem 4.6 with Theorem 4.5 now yields the following:

Corollary 4.7. The Weak Additivity Conjecture implies the Collapse Conjecture: $\mathrm{QMA}(k)=\mathrm{QMA}(2)$ for all $k \geq 2$. 


\section{The Power of Unentanglement}

\subsection{Symmetric $Q M A(k)$}

Define the complexity class $\operatorname{SymQMA}(k, a, b)$ the same way as QMA $(k, a, b)$, except that now we are promised that the $k$ witnesses are all identical (in both the completeness and soundness cases). We saw in Section 3.3 that symmetric QMA $(k)$ protocols are sometimes easier to analyze than non-symmetric ones. However, we will now show that assuming the Weak Additivity Conjecture, QMA $(k)$ and SymQMA $(k)$ are actually equivalent. ${ }^{12}$

The first step is to show they are (unconditionally) equivalent up to a loss in error bounds.

Lemma 4.8. $\mathrm{QMA}(k, a, b) \subseteq \operatorname{SymQMA}(k, a, b) \subseteq \mathrm{QMA}\left(k, 1-\frac{(b-a)^{2}}{8 k}, 1-2^{-n}\right)$.

Proof. For the first containment, have each Merlin in the SymQMA protocol send $k$ witnesses (for a total of $k^{2}$ witnesses). Then simulate the QMA protocol by using the $i^{\text {th }}$ witness from the $i^{\text {th }}$ Merlin for all $i \in[k]$. For the second containment, first observe that

$$
\operatorname{SymQMA}(k, a, b) \subseteq \operatorname{SymQMA}\left(k, 1-(b-a), 1-2^{-n}\right),
$$

completely analogously to Lemma 2.5 . Let $\delta=b-a$. Then to simulate a SymQMA $\left(k, 1-\delta, 1-2^{-n}\right)$ protocol without the symmetry promise we do the following. Let $\left|\varphi_{i}\right\rangle$ be the witness sent by the $i^{\text {th }}$ Merlin. Then

- With $1 / 2$ probability, Arthur performs a swap test between $\left|\varphi_{1}\right\rangle$ and a random other witness, and accepts if and only if the swap test accepts.

- With 1/2 probability, Arthur runs the SymQMA protocol as if the witnesses were identical.

In the completeness case, it is clear that Arthur accepts with probability greater than $1-2^{-n}$. To show soundness we consider two cases, just like in Theorem 4.6. Let $|\Phi\rangle=\left|\varphi_{1}\right\rangle \otimes \cdots \otimes\left|\varphi_{k}\right\rangle$. First suppose $|\Phi\rangle$ is $\varepsilon$-close in trace distance to $\left|\varphi_{1}\right\rangle^{\otimes k}$. Then by Proposition 2.6, when he runs the SymQMA protocol Arthur will reject with probability at least $\delta-\varepsilon$. Next suppose $|\Phi\rangle$ is $\varepsilon$-far from $\left|\varphi_{1}\right\rangle^{\otimes k}$. Then $\left|\left\langle\left.\varphi_{1}\right|^{\otimes k} \mid \Phi\right\rangle\right|^{2}<1-\varepsilon^{2}$ by Proposition 2.9. So by Proposition 2.8, we have

$$
\sum_{i=1}^{k}\left(1-\left|\left\langle\varphi_{1} \mid \varphi_{i}\right\rangle\right|^{2}\right)>\varepsilon^{2} .
$$

Hence when Arthur performs a swap test, he rejects with probability greater than $\varepsilon^{2} /(2 k)$.

Setting $\varepsilon:=3 \delta / 4$, we thus find that the protocol rejects with probability at least $\delta^{2} /(8 k)$.

Combining Lemma 4.8 with Theorem 4.6, we immediately get the following.

Theorem 4.9. The $\mathrm{QMA}(2)$ amplification conjecture implies $\operatorname{Sym} \mathrm{QMA}(k)=\mathrm{QMA}(k)=\mathrm{QMA}(2)$ for all $k \geq 2$.

\footnotetext{
${ }^{12}$ This does not mean that proving the Weak Additivity Conjecture would supersede the analysis of the non-symmetric case in Section 3. Our result will only show that, assuming the Weak Additivity Conjecture, the witness sizes in QMA and SymQMA protocols are polynomially related, which is vacuous in the context of our 3S AT protocol.
} 


\section{Nonexistence of perfect disentanglers}

In this section, we will speak interchangeably about Hilbert spaces and spaces of density operators over those Hilbert spaces.

Definition 5.1. Let $\mathcal{H}$ and $\mathcal{K}$ be two finite-dimensional Hilbert spaces. Then given a superoperator $\Phi: \mathcal{H} \rightarrow \mathcal{K} \otimes \mathcal{K}$, we say $\Phi$ is an $(\varepsilon, \delta)$-disentangler if

(i) $\Phi(\rho)$ is $\varepsilon$-close to a separable state for every $\rho$, and

(ii) for every separable state $\sigma$, there exists a $\rho$ such that $\Phi(\rho)$ is $\delta$-close to $\sigma$.

As pointed out in Section 1.5, if for sufficiently small constants $\varepsilon, \delta$ there exists an $(\varepsilon, \delta)$ disentangler with $\log \operatorname{dim} \mathcal{H}=\operatorname{poly}(\log \operatorname{dim} \mathcal{K})$ —and if, moreover, that disentangler can be implemented in quantum polynomial time- then $\mathrm{QMA}(2)=\mathrm{QMA}$.

Watrous (personal communication) has proposed the following fundamental conjecture.

Conjecture 5.2 (Watrous). For all constants $\varepsilon, \delta<1$, any $(\varepsilon, \delta)$-disentangler requires

$$
\operatorname{dim} \mathcal{H}=2^{\Omega(\operatorname{dim} \mathcal{K})} .
$$

A proof of Conjecture 5.2 would be an important piece of formal evidence that $\mathrm{QMA}(2) \neq \mathrm{QMA}$, and might even lead to a "quantum oracle separation" (as defined by Aaronson and Kuperberg [1]) between the two classes.

Here we show that, at least in the case $\varepsilon=\delta=0$, no disentangler exists in any finite dimension. The counterexamples in Section 1.5 imply that this result would be false if we let either $\varepsilon$ or $\delta$ be nonzero.

Theorem 5.3. Let $\Phi: \mathcal{H} \rightarrow \mathcal{K} \otimes \mathcal{K}$ be any superoperator whose image is the set of separable states. Then $\operatorname{dim} \mathcal{K} \geq 2$ implies $\operatorname{dim} \mathcal{H}=\infty$.

Proof. For any pure state $|\alpha\rangle \in \mathcal{K}$, by assumption there exists a state $\rho_{\alpha}$ such that $\Phi\left(\rho_{\alpha}\right)=|\alpha\rangle\langle\alpha| \otimes$ $|\alpha\rangle\langle\alpha|$. By convexity, we can assume $\rho_{\alpha}=\left|\phi_{\alpha}\right\rangle\left\langle\phi_{\alpha}\right|$ is pure. Also, suppose $\operatorname{dim} \mathcal{H}$ is finite. Then $\Phi$ admits an operator-sum representation $\Phi(\rho)=\sum_{i=1}^{k} E_{i} \rho E_{i}^{\dagger}$ where $\sum_{i=1}^{k} E_{i}^{\dagger} E_{i}=I$. We then have

$$
\Phi\left(\left|\phi_{\alpha}\right\rangle\left\langle\phi_{\alpha}\right|\right)=\sum_{i=1}^{k} E_{i}\left|\phi_{\alpha}\right\rangle\left\langle\phi_{\alpha}\left|E_{i}^{\dagger}=\right| \alpha\right\rangle\langle\alpha|\otimes| \alpha\rangle\langle\alpha| .
$$

So again by convexity, we find that $E_{i}\left|\phi_{\alpha}\right\rangle$ must be a multiple of $|\alpha\rangle|\alpha\rangle$ for all $i$ and $\alpha$; that is, there exist constants $c_{\alpha, i}$ such that $E_{i}\left|\phi_{\alpha}\right\rangle=c_{\alpha, i}|\alpha\rangle|\alpha\rangle$.

Now let $|\alpha\rangle,|\beta\rangle$ be any two pure states in $\mathcal{K}$ with $|\alpha\rangle \neq|\beta\rangle$. Also let $|\psi\rangle=a\left|\phi_{\alpha}\right\rangle+b\left|\phi_{\beta}\right\rangle$ for some nonzero real numbers $a, b$. Then

$$
\begin{aligned}
\Phi(|\psi\rangle\langle\psi|) & =a^{2} \Phi\left(\left|\phi_{\alpha}\right\rangle\left\langle\phi_{\alpha}\right|\right)+b^{2} \Phi\left(\left|\phi_{\beta}\right\rangle\left\langle\phi_{\beta}\right|\right)+a b \Phi\left(\left|\phi_{\alpha}\right\rangle\left\langle\phi_{\beta}\right|\right)+a b \Phi\left(\left|\phi_{\beta}\right\rangle\left\langle\phi_{\alpha}\right|\right) \\
& =a^{2}|\alpha\rangle\langle\alpha|\otimes| \alpha\rangle\left\langle\alpha\left|+b^{2}\right| \beta\right\rangle\langle\beta|\otimes| \beta\rangle\langle\beta|+a b c| \alpha\rangle\langle\beta|\otimes| \alpha\rangle\langle\beta|+a b \bar{c}| \beta\rangle\langle\alpha|\otimes| \beta\rangle\langle\alpha|,
\end{aligned}
$$


where

$$
c=\sum_{i=1}^{k} c_{\alpha, i} \bar{c}_{\beta, i}
$$

We claim that $c=0$. To see this, recall that $\Phi(|\psi\rangle\langle\psi|)$ is a separable mixed state, and consider any decomposition of $\Phi(|\psi\rangle\langle\psi|)$ into separable pure states. Since $\Phi(|\psi\rangle\langle\psi|)$ is a mixed state in the subspace spanned by $|\alpha\rangle|\alpha\rangle$ and $|\beta\rangle|\beta\rangle$, every pure state in the support of $\Phi(|\psi\rangle\langle\psi|)$ must have the form $x|\alpha\rangle|\alpha\rangle+y|\beta\rangle|\beta\rangle$. But by the assumption $|\alpha\rangle \neq|\beta\rangle$, such a state cannot be separable unless $x=0$ or $y=0$. Hence the only separable pure states in the support of $\Phi(|\psi\rangle\langle\psi|)$ are $|\alpha\rangle|\alpha\rangle$ and $|\beta\rangle|\beta\rangle$. Therefore $a b c=0$. But $a$ and $b$ were nonzero, so $c=0$ as claimed.

This means in particular that $\Phi\left(\left|\phi_{\alpha}\right\rangle\left\langle\phi_{\beta}\right|\right)=0$ for all $|\alpha\rangle \neq|\beta\rangle$. Hence

$$
\left\langle\phi_{\beta} \mid \phi_{\alpha}\right\rangle=\sum_{i=1}^{k}\left\langle\phi_{\beta}\left|E_{i}^{\dagger} E_{i}\right| \phi_{\alpha}\right\rangle=\operatorname{Tr}\left(\sum_{i=1}^{k} E_{i}\left|\phi_{\alpha}\right\rangle\left\langle\phi_{\beta}\right| E_{i}^{\dagger}\right)=\operatorname{Tr}\left(\Phi\left(\left|\phi_{\alpha}\right\rangle\left\langle\phi_{\beta}\right|\right)\right)=0 .
$$

So for different $|\alpha\rangle$ 's, the states $\left|\phi_{\alpha}\right\rangle$ are all orthogonal, and since the number of $|\alpha\rangle$ 's is infinite, dim $\mathcal{H}$ must be infinite as well.

\section{Open problems}

\subsection{The power of multiple merlins}

The power of QMA(2) and related classes is still poorly understood. Can we find a "classical" problem (for example, a group-theoretic problem like those of Watrous [29]) that is in QMA(2) but not obviously in QMA? Can we find a natural QMA $(k)$-complete promise problem?

We still do not have any upper bound on QMA(2) better than the trivial NEXP, or even good evidence for such an upper bound. As mentioned before, an earlier version of this paper showed that QMA(2) $\subseteq$ PSPACE assuming the "Strong Amplification Conjecture," but Fernando Brandão (personal communication) subsequently showed that the same conjecture implies $Q M A(2)=Q M A$. Can we show under a plausible conjecture that $\mathrm{QMA}(2) \subseteq \mathrm{EXP}$ or (better yet) $\mathrm{QMA}(2) \subseteq \mathrm{PSPACE}$ ?

Regarding our 3SAT protocol, can we reduce the number of provers to two? Can we reduce the number of qubits below $\widetilde{O}(\sqrt{n})$, or alternatively, give some sort of evidence against this possibility? For example, can we show that $\Omega(\sqrt{n})$ qubits are information-theoretically required for the Uniformity Test? Also, can we show that a $\mathrm{QMA}(k)$ protocol for $3 \mathrm{~S}$ AT with $n^{o(1)}$ qubits would have unlikely complexity consequences?

A long-shot possibility would be to give a quantum algorithm to find the unentangled witnesses in the $3 \mathrm{SAT}$ protocol, in as much time as it would take were the witnesses entangled. This would yield a $2^{\widetilde{O}(\sqrt{n})}$-time quantum algorithm for $3 \mathrm{SAT}$.

\subsection{Amplification and other complexity issues}

In defining QMA $(k)$, does it matter if the amplitudes are reals or complex numbers? For BQP and QMA, it is not hard to show that this distinction is irrelevant. Interestingly, though, the usual equivalence proofs 


\section{S. Aaronson, S. Beigi, A. Drucker, B. Fefferman, And P. Shor}

break down for $\mathrm{QMA}(k)$. As evidence that $\mathrm{QMA}(k)$ might actually be sensitive to the difference between reals and complex numbers, consider the analysis of our 3SAT protocol: in Section 3.4, the result that we need becomes much simpler to prove when we assume all amplitudes are real.

Can we show directly (i.e., without proving the Weak Additivity Conjecture) that

$$
\mathrm{QMA}(k)=\mathrm{QMA}(2) \text {, }
$$

or that $\mathrm{QMA}(2)$ protocols can be amplified?

Can we prove Conjecture 5.2: that there is no $(\varepsilon, \delta)$-disentangler with poly $(n)$ qubits and $\varepsilon, \delta>0$ ? Can we at least rule out such a disentangler when either $\varepsilon>0$ or $\delta>0$ ? Related to that, can we give a quantum oracle $U$ (as defined by Aaronson and Kuperberg [1]) such that $\mathrm{QMA}^{U} \neq \mathrm{QMA}^{U}(2)$ ? Can we at least show that Conjecture 5.2 would imply the existence of such an oracle?

Define the complexity class QMA $(2 ; h)$ to be the same as QMA(2), except that now, instead of being completely unentangled, the two Merlins are allowed to share $h$ EPR pairs $\frac{1}{\sqrt{2}}(|00\rangle+|11\rangle) .{ }^{13}$ Can we show-perhaps under some assumption—that QMA $(2 ; h)=\mathrm{QMA}(2)$ for all polynomial $h$ ?

\section{3 $\mathrm{QMA}(k)$ with unentangled measurements}

Recall that our 3SAT protocol involved three tests: Satisfiability, Symmetry, and Uniformity. Suppose we are willing to settle for completeness $1-\varepsilon$ rather than 1 , and suppose we modify the Uniformity Test so that Arthur rejects on not seeing enough collisions. Then can the Symmetry Test be omitted? If so, then the resulting protocol would have the extremely interesting property of making no entangled measurements, yet nevertheless depending crucially on the absence of entanglement among the witnesses.

More generally, define $\operatorname{BellQMA}(k)$ to be the subclass of $\mathrm{QMA}(k)$ in which Arthur is restricted to making a separate measurement on each witness $\left|\varphi_{i}\right\rangle$, with no entanglement between the measurements, and no measurement depending on the outcome of any other. (The name arises because Arthur is essentially restricted to performing a "Bell experiment.") In an earlier version of this paper, we raised the question of whether $\operatorname{BellQMA}(k)=\mathrm{QMA}$ : that is, whether a QMA $(k)$ protocol with separate measurements on each witness ever does superpolynomially better than an ordinary QMA protocol. Recently, Brandão [9] managed to settle our question, showing that $\operatorname{BellQMA}(k)=\mathrm{QMA}$ for all constant $k \cdot{ }^{14}$ Indeed, Brandão's result applies even to the stronger model in which Arthur can first measure $\left|\varphi_{1}\right\rangle$, then choose a measurement for $\left|\varphi_{2}\right\rangle$ depending on the outcome of the $\left|\varphi_{1}\right\rangle$ measurement, and so on up to $\left|\varphi_{k}\right\rangle$. (In other words, in which there is one-way communication from earlier to later measurement steps.)

On the other hand, define LOCCQMA(k) to be the subclass of QMA $(k)$ in which Arthur's measurements on the $k$ witnesses must be unentangled, but can involve arbitrary local operations and classical communication. (In other words, Arthur can perform a partial measurement on $\left|\varphi_{1}\right\rangle$, then based on the outcome of that measurement perform a partial measurement on $\left|\varphi_{2}\right\rangle$, then based on the outcome of that measurement perform another partial measurement on $\left|\varphi_{1}\right\rangle$, and so on.) Then where LOCCQMA(k)

\footnotetext{
${ }^{13}$ A similar notion was considered by Gavinsky [12]. By contrast, Kobayashi and Matsumoto [18] allow two provers to share an arbitrary polynomial amount of entanglement.

${ }^{14}$ The case of larger $k$ remains open, and therefore Brandão's result has no implications for the earlier question about our 3S AT protocol.
} 
sits between $\mathrm{QMA}$ and $\mathrm{QMA}(k)$ remains an open problem. (Note that it is trivial to show amplification for $\operatorname{LOCCQMA}(k)$, as for $\operatorname{BellQMA}(k)$. This is because, without entangling measurements, the entanglement-swapping problem described in Section 1.4 can never arise.)

\section{Acknowledgments}

We thank Fernando Brandão for pointing out that our "Strong Amplification Conjecture" implies not only $\mathrm{QMA}(2) \subseteq$ PSPACE but $\mathrm{QMA}(2)=\mathrm{QMA}$, and that our original version of Lemma 4.3 contained a bug, among other extremely helpful comments; Norbert Schuch for pointing out that Theorem 4.5 can be based on a conjecture about entanglement of formation rather than squashed entanglement; Madhu Sudan for pointers on the PCP Theorem; John Watrous for posing Conjecture 5.2; an anonymous reviewer for greatly simplifying the proof of Lemma 4.3 and other help; and Patrick Hayden, Ryan O'Donnell, Alain Tapp, and Andreas Winter for helpful discussions.

\section{References}

[1] S. Aaronson And G. Kuperberg: Quantum versus classical proofs and advice. Theory of Computing, 3(7):129-157, 2007. Previous version in Proc. CCC 2007 and quant-ph/0604056. [ToC:v003/a007, arXiv:quant-ph/0604056]. 36, 38

[2] D. Aharonov And T. Naveh: Quantum NP - a survey. quant-ph/0210077, 2002. [arXiv:quant$\mathrm{ph} / 0210077] .2$

[3] M. Ben-Or, S. Goldwasser, J. Kilian, and A. Wigderson: Multi-prover interactive proofs: how to remove the intractability assumptions. In Proc. 20th STOC, pp. 113-131, New York, NY, USA, 1988. ACM Press. [STOC:62212.62223]. 2

[4] C. Bennett, E. Bernstein, G. Brassard, And U. Vazirani: Strengths and weaknesses of quantum computing. SIAM J. Comput., 26(5):1510-1523, 1997. [SICOMP:10.1137/S0097539796300933, arXiv:quant-ph/9701001]. 28, 29

[5] C. H. Bennett, G. Brassard, C. Crépeau, R. Jozsa, A. Peres, and W. Wootters: Teleporting an unknown quantum state by dual classical and EPR channels. Phys. Rev. Lett., 70:1895-1898, 1993. [PRL:10.1103/PhysRevLett.70.1895]. 7

[6] C. H. Bennett, D. P. DiVincenzo, J. A. Smolin, And W. K. Wootters: Mixedstate entanglement and quantum error correction. Phys. Rev. A, 54(5):3824-3851, 1996. [PRA:10.1103/PhysRevA.54.3824, arXiv:quant-ph/9604024]. 7, 29

[7] H. BLIER AND A. TAPP: All languages in NP have very short quantum proofs. arXiv:0709.0738, 2007. [arXiv:0709.0738]. 2, 3

[8] D. M. Bloom And W. Knight: A birthday problem. Amer. Math. Monthly, 80(10):1141-1142, December 1973. 14 
[9] F. BRandẽo: Entanglement Theory and the Quantum Simulation of Many-Body Physics. PhD thesis, Imperial College, London, 2008. [arXiv:0810.0026]. 38

[10] M. Christandl And A. Winter: "Squashed entanglement" - an additive entanglement measure. J. Math. Phys., 45(3):829-840, 2004. [arXiv:quant-ph/0308088]. 7, 31

[11] I. DINUR: The PCP theorem by gap amplification. J. ACM, 54(3):12, 2007. [JACM:1236457.1236459]. 3, 5, 12, 28

[12] D. Gavinsky: On the role of shared entanglement. Quantum Inf. Comput., 8(1-2):82-95, 2008. [arXiv:quant-ph/0604052]. 38

[13] M. B. Hastings: A counterexample to additivity of minimum output entropy. arXiv:0809.3972, 2008. [arXiv:quant-ph/0809.3972]. 3, 7, 31

[14] S. Khanna, M. Sudan, L. Trevisan, and D. P. Williamson: The approximability of constraint satisfaction problems. SIAM J. Comput., 30(6):1863-1920, 2000. [SICOMP:10.1137/S0097539799349948]. 12

[15] A. Kitaev, A. Shen, And M. N. Vyalyi: Classical and Quantum Computation. American Mathematical Society, 2002. 2

[16] H. Klauck, A. NAyak, A. TA-Shma, And D. Zuckerman: Interaction in quantum communication. IEEE Trans. Inform. Theory, 53(6):1970-1982, 2007. Earlier version in STOC'2001 and quant-ph/0603135. [doi:10.1109/TIT.2007.896888]. 30

[17] E. KNILL: Quantum randomness and nondeterminism. quant-ph/9610012, 1996. [arXiv:quantph/9610012]. 2

[18] H. Kobayashi and K. Matsumoto: Quantum multi-prover interactive proof systems with limited prior entanglement. J. Comput. System Sci., 66(3):429-450, 2003. [JCSS:10.1016/S00220000(03)00035-7]. 38

[19] H. Kobayashi, K. Matsumoto, and T. Yamakami: Quantum Merlin-Arthur proof systems: are multiple Merlins more helpful to Arthur? In Proc. 14th Intern. Symp. Algorithms and Computation, volume 2906 of LNCS, pp. 189-198, 2003. [ISAAC:847uk09hm42tdu3q, arXiv:quantph/0306051]. 2, 10

[20] R. König And R. Renner: A de Finetti representation for finite symmetric quantum states. $J$. Math. Phys., 46(122108), 2005. quant-ph/0410229. [arXiv:quant-ph/0410229]. 7, 8

[21] Y.-K. LiU, M. Christandl, And F. Verstraete: Quantum computational complexity of the N-representability problem: QMA complete. Phys. Rev. Lett., 98(11), 2007. [PRL:10.1103/PhysRevLett.98.110503, arXiv:quant-ph/0609125]. 2, 8

[22] C. Marriott And J. Watrous: Quantum Arthur-Merlin games. Comput. Complexity, 14(2):122-152, 2005. [CC:h0521k6666871652]. 9 
[23] M. Nielsen And I. ChuAng: Quantum Computation and Quantum Information. Cambridge University Press, 2000. 10, 30

[24] M. Ohya AND D. PeTZ: Quantum Entropy and its Use. Springer, 1993. 30

[25] C. H. PAPAdimitriou AND M. H. YANNAKAKIS: Optimization, approximation, and complexity classes. J. Comput. System Sci., 43(3):425-440, 1991. [JCSS:10.1016/0022-0000(91)90023-X]. 12

[26] R. RAZ: A parallel repetition theorem. SIAM J. Comput., 27(3):763-803, 1998. Earlier version in STOC 1995. [SICOMP:10.1137/S0097539795280895]. 2

[27] P. W. SHOR: Equivalence of additivity questions in quantum information theory. Comm. Math. Phys., 246(3):453-472, 2004. [Springer:a6d2vegprhlqvrby, arXiv:quant-ph/0305035]. 7, 31

[28] V. Vedral and M. B. Plenio: Entanglement measures and purification procedures. Phys. Rev. A, 57(3):1619-1633, 1998. [PRA:10.1103/PhysRevA.57.1619, arXiv:quant-ph/9707035]. 30

[29] J. Watrous: Succinct quantum proofs for properties of finite groups. In Proc. 41st FOCS, pp. 537-546, Silver Spring, MD, USA, 2000. IEEE Computer Society Press. [doi:10.1109/SFCS.2000.892141]. 2, 37

[30] M. Zukowski, A. Zeilinger, M. A. Horne, And A. K. Ekert: "event-ready-detectors": Bell experiment via entanglement swapping. Phys. Rev. Lett., 71(26):4287-4290, 1993. [PRL:10.1103/PhysRevLett.71.4287]. 6

\section{AUTHORS}

Scott Aaronson

assistant professor

EECS Department

Massachusetts Institute of Technology, Cambridge, MA

aaronson@csail.mit.edu

http://www.scottaaronson.com

Salman Beigi

graduate student

Department of Mathematics

Massachusetts Institute of Technology, Cambridge, MA

salman@mit.edu

http://web.mit.edu/salman/www/ 
Andrew Drucker

graduate student

EECS Department

Massachusetts Institute of Technology, Cambridge, MA

add3993@yahoo.com

http://andysresearch.blogspot.com/

Bill Fefferman

graduate student

Department of Computer Science

California Institute of Technology, Pasadena, CA

wjf@caltech.edu

http://www.its.caltech.edu/ $\sim_{\text {wjf }}$

Peter Shor

professor

Massachusetts Institute of Technology, Cambridge, MA

shor@math.mit.edu

http://www-math.mit.edu/ shor/

\section{ABOUT THE AUTHORS}

SCOTT AARONSON is a theoretical computer scientist and blogger. This is his fourth paper in Theory of Computing.

Salman BeIGI received his B. S. at Sharif University of Technology, Tehran in 2004. He is currently finishing his Ph. D. at the MIT Math Department under the direction of Peter Shor. The title of his thesis is "Quantum Proof Systems and Entanglement Theory." He will continue his research as a postdoc at the Institute for Quantum Information at Caltech. His interests include quantum complexity theory, quantum coding theory, photography, and playing daf, a traditional Persian musical instrument.

ANDREW DRUCKER is a Ph. D. student in theoretical computer science at MIT, supervised by Scott Aaronson. He has broad interests in complexity theory, and also enjoys running, live jazz, and the game of Go.

BILl FEFFERMAN is a Ph. D. student at Caltech in computer science and at the Institute for Quantum Information. He started this research while visiting MIT, and continued it at the University of Chicago, where he was an undergraduate. His research interests are quantum computing and computational complexity.

PETER SHOR is a professor at MIT. He is known for his factoring algorithm. 